\title{
Amenability, Poincaré Series and Quasiconformal Maps
}

\section{Citation}

McMullen, Curtis T. 1989. Amenability, Poincare series and quasiconformal maps. Inventiones Mathematicae 97(1): 95-127.

\section{Published Version}

doi:10.1007/BF01850656

\section{Permanent link}

http://nrs.harvard.edu/urn-3:HUL.InstRepos:3446032

\section{Terms of Use}

This article was downloaded from Harvard University's DASH repository, and is made available under the terms and conditions applicable to Other Posted Material, as set forth at http:// nrs.harvard.edu/urn-3:HUL.InstRepos:dash.current.terms-of-use\#LAA

\section{Share Your Story}

The Harvard community has made this article openly available.

Please share how this access benefits you. Submit a story.

Accessibility 


\title{
Amenability, Poincaré series and quasiconformal maps
}

\section{Curt McMullen *}

Department of Mathematics, Princeton University, Princeton NJ 08544, USA

\begin{abstract}
Summary. Any covering $Y \rightarrow X$ of a hyperbolic Riemann surface $X$ of finite area determines an inclusion of Teichmüller spaces Teich $(X) \hookrightarrow T e i c h(Y)$. We show this map is an isometry for the Teichmüller metric if the covering is amenable, and contracting otherwise. In particular, we establish $\|\Theta\|<1$ for classical Poincaré series (Kra's 'Theta conjecture').

The appendix develops the theory of geometric limits of quadratic differentials, used in this paper and a sequel.
\end{abstract}

\section{Introduction: The $\Theta$ operator}

Definitions. Let $X$ be a Riemann surface. $X$ is hyperbolic if it is covered by the unit disk $\Delta$, and $X$ is of finite type if it is obtained from a compact surface by deleting a finite number of points.

Let $Q(X)$ denote the space of holomorphic quadratic differentials $\phi(z) d z^{2}$ on $X$, such that

$$
\|\phi\|=\int_{X}|\phi(z)||d z|^{2}<\infty .
$$

With the above norm, $Q(X)$ is a Banach space. Let $B_{X}=\{\phi:\|\phi\|<1\}$ denote the open unit ball in $Q(X)$.

Now suppose $f: Y \rightarrow X$ is a covering map. Then there is a natural operator

$$
\Theta: Q(Y) \rightarrow Q(X)
$$

(pull-back over branches of $f^{-1}$ and sum). This operator is similar to pushforward of measures, except that variations in the phase of $\phi$ over different sheets may cause cancellation. Thus $\|\Theta\| \leqq 1$.

In Teichmüller theory, $Q(X)$ is the contangent space to the Teichmüller space of $X$, the norm introduced above is the infinitesimal form of the

* Research partially supported by an NSF Postdoctoral Fellowship 
Teichmüller cometric, and $\Theta$ is the coderivative of the map Teich $(X) \rightarrow$ Teich $(Y)$ induced by the covering $Y \rightarrow X$ (see [Gar]).

$\Theta$ was introduced by Poincaré in the case of the universal covering of a hyperbolic Riemann surface of finite type [Poin].

We will show that $\|\Theta\|<1$ for these classical Poincare series; this is Kra's 'Theta conjecture'.

More generally, say a covering $Y \rightarrow X$ is amenable if there are large balls with small boundary in a graphic caricature of the covering (see $\S \S 2$ and 3.) If $X$ is of finite type, its universal cover is nonamenable.

Theorem 1.1. Let $Y \rightarrow X$ be a covering of a hyperbolic Riemann surface $X$. Either:

1. The covering is amenable, and $\Theta\left(B_{Y}\right)=B_{X}$, or

2. The covering is nonamenable, and $\overline{\Theta\left(B_{Y}\right)}$ is contained in the interior of $B_{X}$.

Corollary 1.2. Suppose in addition $X$ is of finite type. Either

1. The covering is amenable, $\|\Theta\|=1$ and the induced map Teich $(X) \rightarrow$ Teich $(Y)$ is a global isometry for the Teichmüller metric, or

2. The covering is nonamenable, $\|\Theta\|<1$ and $\operatorname{Teich}(X) \rightarrow \operatorname{Teich}(Y)$ is contracting.

The corollary is immediate from finite dimensionality of $Q(X)$, and the necessity and sufficiency of Hamilton's condition for an extremal quasiconformal map (see [Str 2]).

Contraction of the inclusion of Teich $(X)$ into universal Teichmüller space can be restated as follows:

Corollary 1.3. Let $f: X_{1} \rightarrow X_{2}$ be a Teichmüller mapping between hyperbolic Riemann surfaces of finite type. Then the map $f: \Delta \rightarrow \Delta$ obtained by lifting $f$ to the universal covers of $X_{1}$ and $X_{2}$ is not extremal among quasiconformal maps with the same boundary values (unless $f$ is conformal).

\section{Dependence on moduli}

It is useful to understand how the amount of contraction of the inclusion

$$
\operatorname{Teich}(X) \hookrightarrow \operatorname{Teich}(Y)
$$

depends on the geometry of $X$. Using an Euler characteristic argument we obtain:

Theorem 1.4 (Geometric Bound). Let $X$ be a hyperbolic Riemann surface of finite type, $Y \rightarrow X$ an infinite-sheeted covering space with finitely generated fundamental group. Then $\left\|\Theta_{Y / X}\right\|<c(n, L)<1$, where $c(n, L)$ is a continous function of $n=$ the number of generators of $\pi_{1}(Y)$ and $L=$ the length of the shortest geodesic on $X$.

Thus the amount of contraction is controlled by the location of $X$ in moduli space. (However there is no global bound - see $\S 10$ below.) 


\section{Application to 3-manifolds}

A key tool in Thurston's construction of hyperbolic structures on 3-manifolds is the skinning map, an iteration on Teichmüller space whose fixed point yields a geometric structure [Th 1$]$.

The skinning map factors through canonical maps between Teichmüller spaces whose contraction is controlled by a refinement of the geometric bound above (Theorem 11.1). In a sequel [Mc], this refinement is coupled with the theory of geometric limits of quadratic differentials (developed in the Appendix below) to give a new, analytic proof of the existence of a fixed point.

\section{Outline of the paper}

We begin with a sketch of the proof of Theorem 1.1 for a nonamenable covering.

Suppose $\Theta(\phi)=\psi$. To prevent loss of mass, the phase of $\phi$ must nearly agree with that of the pull-back of $\psi$ to $Y$, at least over a region $Y_{0}$ which contains most of the mass of $|\phi|$. But agreement of phase implies the mass distribution of $\phi$ mimics that of $\psi$, which in the large is determined by the combinatorics of the covering $Y \rightarrow X$. For a nonamenable covering, most of the mass of $Y_{0}$ will be near its boundary, where the pairing is inefficient by a definite amount; this forces $\|\Theta\|<1$.

$\S \S 2$ and 3 develop the combinatorial theory of amenable and nonamenable graphs and coverings, and establish the global features of the argument above. Explicit 'rates of nonamenability' are estimated in $\S 4$; these estimates provide many examples of nonamenable coverings and also play a role in the proof of Theorem 1.4 .

$\S 5$ is a study of the shape of the unit ball in $Q(\Delta)$, which justifies the local part of the argument: quadratic differentials with nearly synchronized foliations have similar mass distributions.

In $\S 6$, we formulate a 'fiberwise' version of the statement $\|\Theta\|<1$; this is established in $\S 7$, and we deduce the nonamenable half of Theorem 1.1 in $\S 8$. $\S 9$ treats the case of amenable coverings, completing the proof of Theorem 1.1.

In $\S \S 10$ and 11 we analyze the proof further to obtain the dependence on moduli (Theorem 1.4 and its refinements). The discussion in $\S 10$ draws on classical results in Teichmüller theory, while $\S 11$ employs a geometric limit argument.

Finally $\S 12$ sketches a generalization to Poincaré series for higher order differentials and quotients of bounded domains in $\mathbf{C}^{n}$.

The Appendix develops the theory of geometric limits of quadratic differentials, for use in $\S 11$ and in a sequel on the skinning map [Mc].

Bibliographical remarks. Corollary 1.3 was checked for many examples by Strebel [Str 1]; see Strebel's recent survey [Str 4] for further background on extremal quasiconformal maps. The relation between $\|\Theta\|$ and lifts of Teichmüller mappings is mentioned in [Kra2]. Arithmetical aspects of $\|\Theta\|$ were explored by 
Parson and Sheingorn [PS 1], [PS 2]. Interactions between function theory, covering spaces and amenability also appear in papers of Brooks $[\mathrm{Br}]$ and Lyons and Sullivan [LS].

We have recently learned that Ohtake proved $\|\Theta\|=1$ for abelian coverings $[\mathrm{Oh}]$.

\section{Nonamenable graphs and slowly varying functions}

In this section we will isolate some combinatorial properties of nonamenable groups, and more generally nonamenable graphs. The added generality will allow us to deal with irregular covers.

Definitions. Let $G$ be a graph. For any set $V$ of vertices of $G$, define $b(V)$, the border of $V$, to be the set of vertices at distance 1 from $V$. (A vertex at distance 1 is connected to $V$ by an edge but does not itself lie in $V$ ).

Define the expansion $\gamma$ of $G$ as the infimum of $|b(V)| /|V|$ as $V$ ranges over all finite sets of vertices. If the expansion is $0, G$ is amenable. Otherwise $G$ is nonamenable, and the size of the border of any set of vertices is a definite fraction of the size of the set itself.

For example: an infinite tree in which every vertex has degree $d$ is amenable for $d=2$, but nonamenable, with expansion $\gamma=(d-2)$, when $d$ is 3 or more.

Now let $f: V \rightarrow \mathbf{R}$ be a positive integrable function on a subset $V$ of the vertices of $G$ (i.e. $\sum_{x \in V} f(x)<\infty$.) For $x$ in the border $b(V)$, define

$$
F(x)=\sup \{f(y): y \text { is adjacent to } x .\}
$$

Theorem 2.1. Suppose that $G$ is nonamenable with expansion $\gamma$, and $f$ is slowly varying in the sense that, for some $\lambda, 0<\lambda<1$,

$$
f(x) \geqq \lambda f(y)
$$

whenever $x$ and $y$ are adjacent vertices of $V$. Then

$$
\sum_{x \in b(V)} F(x) \geqq(\lambda \gamma+\lambda-1) \sum_{x \in V} f(x) .
$$

Remarks.

1. Of course $f(x) \leqq \lambda^{-1} f(y)$ by symmetry.

2. If $f$ varies sufficiently slowly, a definite fraction of its mass is picked up by $F$. This mass will give a definite contribution to the contraction of $\Theta$ in $\S 7$.

3. Setting $f \equiv 1$ on $V$ and $\lambda=1$ gives $|b(V)| \geqq \gamma|V|$ as a special case.

Proof. For every integer $n$, let $V_{n}$ denote the subset of $V$ on which $f(x) \geqq \lambda^{n}$. Then $V_{n}$ is an increasing sequence of finite sets and $V_{n}$ is empty for $n$ sufficiently negative. 
Let $B_{n}=b\left(V_{n}\right) \cap b(V)$, and let $v_{n}$ and $b_{n}$ denote $\left|V_{n}\right|$ and $\left|B_{n}\right|$ respectively. By the slow variation of $f, b\left(V_{n}\right) \subset B_{n} \sqcup\left(V_{n+1}-V_{n}\right)$, so by nonamenability,

$$
b_{n}+\Delta v_{n} \geqq \gamma v_{n},
$$

where $\Delta v_{n}=v_{n+1}-v_{n}$ is the usual combinatorial derivative. Applying this inequality after integrating by parts (note that $\Delta \lambda^{n}<0$ ), we find

$$
\sum \lambda^{n+1} \Delta b_{n}=-\sum b_{n} \Delta \lambda^{n} \geqq \sum \Delta v_{n} \Delta \lambda^{n}-\gamma v_{n} \Delta \lambda^{n},
$$

where we sum over all integers $n$. Integrating the second term on the right by parts, this can be rewritten

$$
\sum \lambda^{n+1} \Delta b_{n} \geqq(\lambda-1+\lambda \gamma) \sum \lambda^{n} \Delta v_{n} .
$$

Now $f(x)$ is bounded above by $\lambda^{n}$ on $V_{n+1}-V_{n}$, so the sum appearing on the right is an upper bound for $\sum f(x)$; similarly the left hand sum is a lower bound for $\sum F(x)$, establishing the theorem.

In geometric situations, we will deal exclusively with graphs of bounded degree (there is a bound on the number of edges attached to each vertex). A set of vertices $I$ is independent if no two elements of $I$ are joined by an edge.

Proposition 2.2. Let $G$ be a graph of degree at most $d, f: V \rightarrow \mathbf{R}$ a non-negative integrable function on the vertices of $G$. Then there exists a set of independent vertices I such that

$$
\sum_{I} f(x) \geqq \frac{1}{d+1} \sum_{V} f(x)
$$

Proof. Sort the vertices of $G$ so $f(x)$ is descending $\left(f\left(x_{0}\right) \geqq f\left(x_{1}\right) \geqq \ldots\right)$, and construct $I$ by a greedy algorithm: recursively add to $I$ the next vertex in sequence which is not adjacent to those already chosen. Then every vertex $y$ not in $I$ can be assigned to an adjacent vertex $x$ in $I$ so $f(x) \geqq f(y)$. This assignment is at most $d$-to-1, and the proposition follows.

\section{From coverings to graphs}

In this section we define (in several equivalent ways) the notion of an amenable, but possibly irregular covering. ([Gre] is a concise reference for the theory of amenable groups, of which this material is a generalization; see also [Pier].)

All manifolds considered are assumed smooth and connected. Let $X$ be a manifold, $p: Y \rightarrow X$ a covering, $p_{*}: \pi_{1}(Y) \subset \pi_{1}(X)$ the induced inclusion of fundamental groups (for our considerations basepoints will be unimportant). 
Definitions. The covering $Y \rightarrow X$ is amenable if there is a linear functional

$$
m: L^{\infty}\left(\pi_{1}(X) / \pi_{1}(Y)\right) \rightarrow \mathbf{R},
$$

invariant under the left-action of $\pi_{1}(X)$, such that $\inf (f) \leqq m(f) \leqq \sup (f)$ for all $f$.

Such a functional $m$ is an invariant mean for the group action.

A group $G$ is amenable if $L^{\infty}(G)$ admits a $G$-invariant mean. Thus, amenability of a regular covering is equivalent to amenability of its group of deck transformations.

Examples.

1. Solvable groups are amenable, as are subgroups and quotient groups of amenable groups.

2. The free group on 2 or more generators is nonamenable.

3. Any nonelementary Fuchsian group contains a free group on 2 generators, and hence is nonamenable.

4. In particular, the fundamental group of hyperbolic Riemann surface of finite type is nonamenable.

5. Subexponential growth implies amenability, but there are amenable groups with exponential growth - such as the solvable group $\left\langle x, y: x y=y^{2} x\right\rangle$.

Using graphs, one can give a more combinatorial characterization of an amenable covering.

Assume for the moment that $\pi_{1}(X)$ has a finite generating set $\mathscr{G}$. From this data, construct the coset graph as follows: take the cosets $\pi_{1}(X) / \pi_{1}(Y)$ as vertices, and connect $x \pi_{1}(Y)$ and $y \pi_{1}(Y)$ by an edge if there exists a $g$ in $\mathscr{G}$ such that $g x \pi_{1}(Y)=y \pi_{1}(Y)$. This graph is combinatorial model for the covering.

It will be useful to introduce finer models, as follows. A net $\mathscr{E}$ on $X$ is a collection of simply connected open sets, whose union $U$ is connected, such that $E \cap E^{\prime}$ is either empty or connected for any two members of $\mathscr{E}$.

To $\mathscr{E}$ we associate its $\breve{C} e c h$ graph, whose vertices are the elements of $\mathscr{E}$ and whose edges join $E$ and $E^{\prime}$ iff $E \cap E^{\prime} \neq \emptyset$. The Čech graph is the same as the 1 -skeleton of the nerve of $\mathscr{E}$.

There is an induced net on $Y$, which we denote by $p^{*}(\mathscr{E})$, whose elements are the components of $p^{-1}(E)$ for each $E$ in $\mathscr{E}$.

Our second combinatorial model for the covering $p: Y \rightarrow X$ is the Čech graph of $p^{*} \mathscr{E}$.

Proposition 3.1. A covering $p: Y \rightarrow X$ is amenable iff one of the following equivalent conditions is satisfied:

1. The coset graph of $K /\left(K \cap \pi_{1}(Y)\right.$ is amenable for every finitely generated subgroup $K$ of $\pi_{1}(X)$.

2. The pullback of the covering to $X_{0}$ is amenable for every submanifold of $X_{0}$ of $X$ with finitely generated fundamental group.

3. The Čech graph of $p^{*} \mathscr{E}$ is amenable for every finite net $\mathscr{E}$ on $X$.

Equivalence of the three conditions is easy to check. That the first condition is equivalent to the existence of an invariant mean on the coset space is essentially Rosenblatt's generalization of Følner's condition [Ros].

As we will never make use of the invariant mean, the reader may also take these combinatorial conditions as the definition of amenability. 


\section{Explicit expansion constants}

Let $Y \rightarrow X$ be a covering of surfaces, $H \hookrightarrow G$ the corresponding inclusion of fundamental groups. Assume both $H$ and $G$ are finitely generated. We will give an estimate for expansion constant $\gamma$ of the coset graph $G / H$ which depends only on Euler characteristics.

This estimate provides many examples of irregular nonamenable coverings, and will also be used to obtain bounds on $\|\Theta\|$ which depend only on the location of $X$ in moduli space $(\S 10)$.

Of course the expansion constant depends on a choice of generating set $\mathscr{G}$ for $G$. Any surface group may be presented as:

$$
\begin{aligned}
& G=\left\langle a_{1}, \ldots, a_{n}\right\rangle \text { or } \\
& G=\left\langle a_{1}, b_{1}, \ldots, a_{g}, b_{g}:\left[a_{1}, b_{1}\right] \ldots\left[a_{g}, b_{g}\right]=1\right\rangle
\end{aligned}
$$

and we will use these generators for $\mathscr{G}$.

The Euler characteristics of $G$ and $H$ are the same as the topological Euler characteristics of $X$ and $Y$. Assume $G$ is nontrivial; then $\chi(G) \leqq 0$.

Lemma 4.1. For any finite set of vertices $V \neq \emptyset$ in the coset graph $G / H$,

$$
|b(V)| \geqq 2(\chi(H)-|V| \chi(G)) .
$$

Proof. The presentation given for $G$ corresponds to a cell complex $C$ which is either a bouquet of circles or a compact surface. There is a covering $\widetilde{C}$ corresponding to $H$, whose 1-skeleton is the coset graph.

Let $V \subset \tilde{C}$ be a finite set of vertices, and let $D$ be the largest subcomplex of $\tilde{C}$ whose 0 -skeleton is $V \cup b(V)$. For the purposes of the estimate, we may assume no component of $\widetilde{C}-D$ is a 2-disk, since otherwise we may include its vertices in $V$ and increase $|V|$ while decreasing $b(V)$. With this assumption, $\chi(D) \geqq \chi(\widetilde{C})=\chi(H)$.

Let $C_{i}, D_{i}$ denote the number of $i$-cells in $C$ and $D$. Noting that the degree of the coset graph (the number of edges incident to each vertex) is $2 C_{1}$, we find:

$$
\begin{aligned}
& D_{0}=|V|+|b(V)|=C_{0}|V|+|b(V)| \\
& D_{1}=\frac{1}{2} \sum_{D_{0}} \operatorname{deg}(v) \geqq \frac{1}{2}\left(2 C_{1}|V|+|b(V)|\right) \\
& D_{2} \leqq|V| C_{2}
\end{aligned}
$$

from which the theorem follows.

Corollary 4.2 (Uniform expansion). Assume $[G: H]=\infty$. Then the expansion of the coset graph satisfies

$$
\gamma \geqq \begin{cases}2|\chi(G)| & \text { if } \chi(H) \geqq 0, \\ |\chi(G) / \chi(H)| & \text { otherwise. }\end{cases}
$$

In particular, if $\chi(G)<0$ the coset graph is nonamenable with expansion bounded below independent of the embedding of $H$ into $G$.

Proof. Combine the preceding Theorem with the estimate $b(V) \geqq 1$, which holds so long as the coset graph is infinite. 
Example. Let $X$ be a hyperbolic Riemann surface of finite type, and let $S \subset X$ be a proper incompressible subsurface. (Incompressible means $\pi_{1}(S)$ injects into $\pi_{1}(X)$; proper means the image is a proper subgroup.) Corresponding to the subgroup $\pi_{1}(S)$ there is an infinite-sheeted covering $Y \rightarrow X$, which is nonamenable
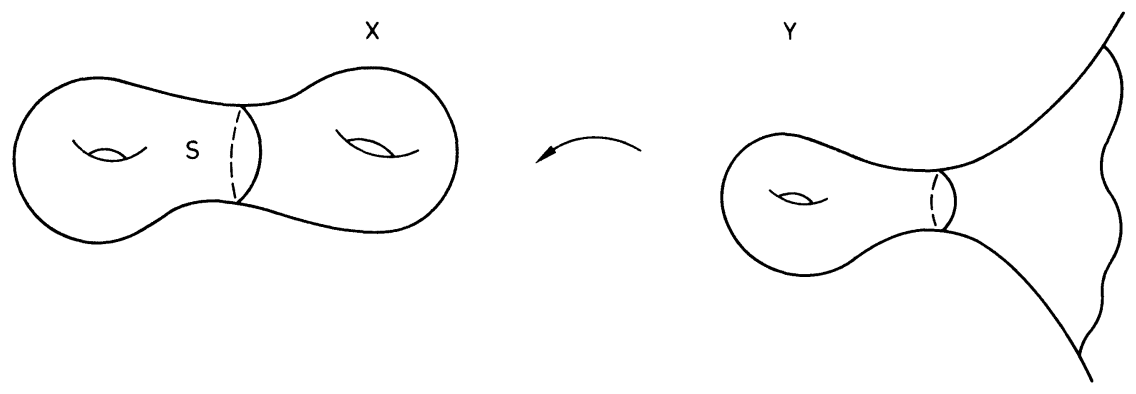

Fig. 1. The covering space of a subsurface

by the result above. Furthermore the expansion is bounded below independent of the choice of $S$, since $\chi(X)<\chi(S) \leqq 0$.

Coverings induced by subsurfaces are said to be geometric. Any covering $Y \rightarrow X$ with $\pi_{1}(Y)$ finitely generated is virtually geometric, i.e. it becomes geometric when pulled back to a finite covering of $X$ [Scott].

\section{Flex of the unit sphere in $Q(\Delta)$}

This section lays the analytic groundwork for the proof of Theorem 1.1.

Let $Q(X)$ be the Banach space of quadratic differentials on a Riemann surface $X$, introduced in the $\S 1$. There is a natural pairing between $Q(X)$ and $M(X)$, the space of measurable Beltrami differentials $\mu(z) d \bar{z} / d z$ with

$$
\|\mu\|=\sup _{x}|\mu(z)|<\infty
$$

namely

$$
\langle\phi, \mu\rangle=\operatorname{Re} \int_{X} \phi(z) \mu(z)|d z|^{2} .
$$

In fact the dual of $Q(X)$ is the quotient of $M(X)$ by the subspace annihilated by $Q$.

Given any $\phi$ of norm $1, \mu=\bar{\phi} /|\phi|$ is the unique element of norm 1 in $M(X)$ such that $\langle\phi, \mu\rangle=1$. Geometrically, this means the unit sphere in $Q(X)$ has no sharp corners - at each point there is a unique supporting hyperplane.

On the unit disk $\Delta$, consider the quadratic differential $\psi(z)=d z^{2} / \pi$. Then $\psi$ is a point on the unit sphere in $Q(\Delta)$. 
Theorem 5.1. Let $\phi$ be an element of the unit sphere in $Q(\Delta)$, such that

$$
\langle\phi, \bar{\psi} /|\psi|\rangle=1-\varepsilon .
$$

Then $\|\psi-\phi\|<O\left(\varepsilon^{\alpha}\right)$ for some fixed exponent $\alpha$.

Remark. The pairing between $\phi$ and $\bar{\psi} /|\psi|$ gives the average, with respect to $|\phi|$-measure, of the cosine of twice the angle between the foliations of $\phi$ and $\psi$. (The foliation of $\psi$ is just the foliation of the disk by horizontal lines.) The theorem says that if the foliations are nearly synchronized, then $\phi$ is close to $\psi$.

Proof. Writing $\|\cdot\|$ and $\langle$,$\rangle in coordinates, our task is to show that$

$$
\int_{\Delta}|\phi(z)|=1
$$

and

together imply

$$
\int_{\Delta} \operatorname{Re} \phi(z)=1-\varepsilon
$$

$$
\int_{\Delta}|1-\phi(z)|=O\left(\varepsilon^{\alpha}\right)
$$

all integrals being with respect to normalized area measure on the disk.

In any measure space, the first two equations imply

$$
\int_{\Delta}|\operatorname{Im} \phi(z)|=O\left(\varepsilon^{1 / 2}\right) .
$$

Since $\operatorname{Im} \phi(z)$ is harmonic, it is controlled by its average over a ball and we obtain

$$
|\operatorname{Im} \phi(z)|=O\left(\varepsilon^{1 / 2} / d(z)^{2}\right),
$$

where $d(z)=d(z, \partial \Delta)$ denotes Euclidean distance from $z$ to the boundary of the disk. Thus $\phi$ maps $B(0,1-d)$ into the strip $\left\{z:|\operatorname{Im} z|<O\left(\varepsilon^{1 / 2} / d^{2}\right)\right\}$.

Similarly $\operatorname{Re} \phi$ is harmonic, so its average over the disk gives the value at the origin: $\operatorname{Re} \phi(0)=1-\varepsilon$. By the Schwarz lemma, most of $B(0,1-d)$ maps near $\phi(0)$, since the image of $B(0,1-d / 2)$ is confined to a narrow strip. Making this precise, one obtains

$$
|1-\phi(z)|=O\left(\varepsilon+\varepsilon^{1 / 2} \log (1 / d(z)) / d(z)^{2}\right) .
$$

Finally we control $|\phi|$ near the boundary of the disk: since $|\phi|$ is subharmonic,

$$
1-\varepsilon \leqq|\phi(0)| \leqq \frac{1}{(1-d)^{2}} \int_{B(0,1-d)}|\phi|
$$

and since the total integral of $|\phi|$ is 1 , this implies

$$
\int_{\{1-d<|z|<1\}}|\phi|=O(d+\varepsilon) .
$$


Combining this with the uniform bound for $|1-\phi(z)|$ on $B(0,1-d)$, one obtains

$$
\int_{\Delta}|1-\phi|=O\left(\varepsilon+d+\varepsilon^{1 / 2} \log (1 / d) / d^{2}\right)=O\left(\varepsilon^{1 / 6} \log (1 / \varepsilon)\right)
$$

if we set $d=\varepsilon^{1 / 6}$. Thus the theorem is true for any exponent $\alpha<1 / 6$.

Remarks.

1. In terms of the geometry of Banach spaces, the theorem states that a point of the unit sphere in $Q(X)$ which is close to the supporting hyperplane for $\psi$ is close to $\psi$ itself; intuitively, the sphere is 'flexed' at $\psi$. If $Q(X)$ were a Hilpert space with its usual norm, the theorem would be true with $\alpha=1 / 2$.

2. Conversely, say a point $p$ of the unit sphere $S$ of a Banach space is flat if for any hyperplane $H$ supporting $S$ at $p$, there exist $\left\langle p_{n}\right\rangle$ of norm 1 whose distance from $H$ tends to 0 but $\left\|p-p_{n}\right\|>\varepsilon>0$. Then many points of the unit sphere $Q(X)$ are flat.

For example, let $\left\langle z_{i}\right\rangle$ be a sequence of points in the disk such that the hyperbolic balls $B\left(z_{i}, r_{i}\right)$ are disjoint and $r_{i} \rightarrow \infty$. Let $M_{i}: \Delta \rightarrow \Delta$ be a sequence of Möbius transformations such that $M_{i}\left(z_{i}\right)=0$, and consider the differential

$$
\alpha=c \sum_{i=1}^{\infty} 2^{-n} M_{i}^{*}(d z)^{2},
$$

where the constant $c$ is chosen to give this expression norm in 1 in $Q(\Delta)$. It is not hard to check that $\left\langle M_{i}^{*}\left(d z^{2} / \pi\right), \bar{\alpha} /|\alpha|\right\rangle \rightarrow 1$ but these differentials do not approach $\alpha$ in the norm topology.

3. There is a close relationship between flexing of the unit sphere and reflexivity. For example, a theorem of Milman asserts that a uniformly convex Banach space is reflexive [Mil]; see also [Pet]. $Q(\Delta)$ is not reflexive, but its unit ball is uniformly convex at certain points, such as $\psi$.

\section{The nonamenable case: selecting the net}

Fix the following data:

- A Riemann surface $X$;

- A quadratic differential $\psi$ in $Q(X)$ with $\|\psi\|=1$; and

- A nonamenable covering $p: Y \rightarrow X$, with induced relative Poincaré operator

$$
\Theta: Q(Y) \rightarrow Q(X) .
$$

For this data, we will establish the following 'fiberwise' version of the Theta Conjecture:

Theorem 6.1 ( $\Theta$ contracts fibers). Let $Q_{\psi}(Y)$ denote the closed subspace of $Q(Y)$ consisting of differentials $\phi$ such that $\Theta(\phi)$ is a multiple of $\psi$. Then $\left\|\Theta \mid Q_{\psi}(Y)\right\|<1$.

To set up the proof, we first construct a net $\mathscr{E}$ on $X$ such that $p^{*} \mathscr{E}$ detects the nonamenability of the covering. 
Recall that $\psi$ gives rises to a flat conformal metric on $X$ with quadratic form $|\psi(z)||d z|^{2}$ (see e.g. [Str3]). At the zeros of $\psi$ the metric has cone-like singularities; these will not concern us.

If $\psi(x) \neq 0$, there is a neighborhood $V$ of $x$ and a conformal isomorphism $g:(V, 0) \rightarrow\left(\Delta_{r}, 0\right)$ such that $\psi \mid V=g^{*}\left(d z^{2}\right)$ (here $\Delta_{r}$ denotes the disk of radius $r$ centered at the origin in $\mathbf{C}$ ). The map $g$ is an isometry from the $\psi$-metric to the Euclidean metric, and $V$ is an embedded $r$-ball in the $\psi$-metric.

Let $r(x)$ denote the injectivity radius of $\psi$ at $x$, i.e. the radius of the largest embedded $r$-ball centered at $x$.

Now choose a finite net $\mathscr{E}$ on $X$ such that, for some fixed $r>0$,

(a) Each element of $\mathscr{E}$ is an embedded $r$-ball in the $\psi$-metric; and

(b) The Cech graph of $p^{*} \mathscr{E}$ is nonamenable.

This is easily done, for example as follows. By nonamenability, there is a finitely generated subgroup $K$ of $\pi_{1}(X)$ whose coset graph is nonamenable. Choose a basepoint at which $\psi \neq 0$ and smooth loops generating this subgroup. The loops may also be chosen to avoid the zeros of $\psi$. By compactness, the injectivity radius of $\psi$ on the loops is bounded below by some $s>0$. Let $\mathscr{E}$ be a finite covering of the union of the loops by embedded $r$-balls, where $r=s / 2$. Any two balls which meet are contained in an embedded $s$-ball, so their intersection is connected, just as in Euclidean space; thus $\mathscr{E}$ is a net. Finally the subgroup $K$ is carried by $\mathscr{E}$, so the Čech graph of $p^{*} \mathscr{E}$ is nonamenable.
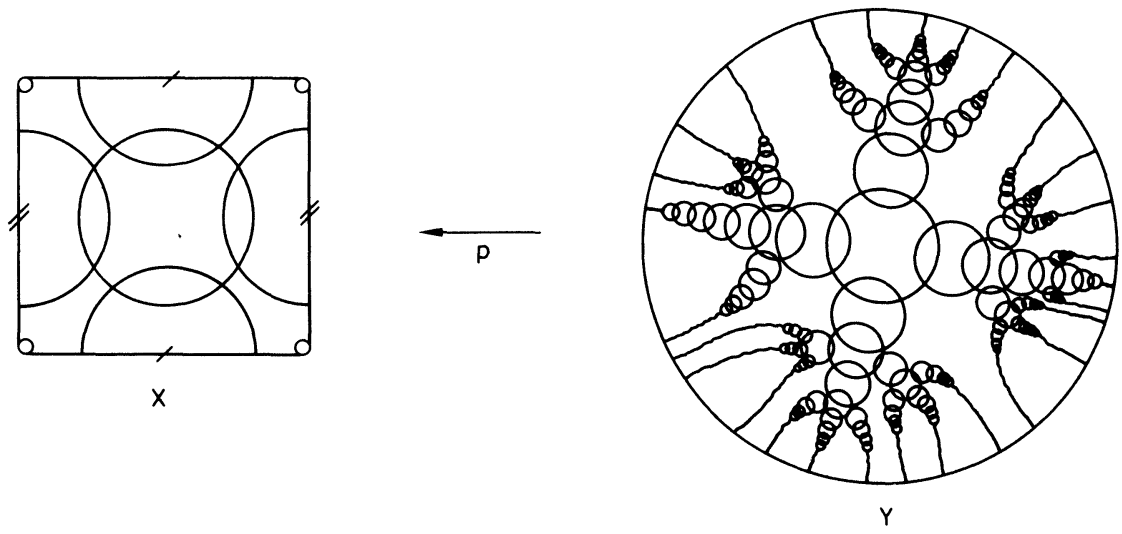

Fig. 2. A net for the punctured torus

For an example in the case of the universal covering of a punctured torus, see Fig. 2. Our bound on $\|\Theta\|$ will depend on the following quantities:

1. The expansion constant of the Čech graph of the induced net $\mathscr{F}=p^{*} \mathscr{E}$;

2. The degree of this graph (which is at most $|\mathscr{E}|)$;

3. The $\psi$-area of $\bigcup \mathscr{E}$; and

4. The minimum overlap between elements of $\mathscr{E}$. This is the minimum fraction of the $\psi$-area of an element $E$ which is shared with an overlapping element $E^{\prime}$. 


\section{Capturing $\|\Theta\|$ fiberwise}

Let $\phi$ be an element of $Q_{\psi}(Y)$ such that $\|\phi\|=1$. To establish Theorem 6.1, we will bound $\|\Theta(\phi)\|$ away from 1 by an amount which is independent of $\phi$. In this section, any bounds written using $O(\cdot)$ will involve constants which are independent of $\phi$.

$\Theta(\phi)=t \psi$ for some constant $t$. Replacing $\phi$ by $t \phi /|t|$ if necessary, we many assume $t \geqq 0$.

Let $\mu=\bar{\psi}|| \psi \mid$ denote the unique element of norm 1 in $M(X)$ such that $\langle\psi, \mu\rangle=1$. For $F$ a measurable subset of $Y$, define the mass and inefficiency of $F$ by:

$$
\begin{aligned}
m(F) & =\int_{F}|\phi|, \\
i(F) & =1-\frac{1}{m(F)} \operatorname{Re} \int_{F} \phi p^{*} \mu .
\end{aligned}
$$

Since $m(Y)=1$,

$$
1-i(Y)=\left\langle\phi, p^{*} \mu\right\rangle=\langle\Theta(\phi), \mu\rangle=\langle t \psi, \mu\rangle=t,
$$

so $i(Y)=1-\|\Theta(\phi)\|$. Our goal is to bound the inefficiency of $Y$ from below.

Mass and inefficiency are always non-negative, so

$$
i(Y)=m(W) i(W)+m(Y-W) i(Y-W) \geqq m(W) i(W),
$$

and hence we need only find a set $W \subset Y$ which has definite mass and inefficiency.

Lemma 7.1. For any $F$ in $\mathscr{F}$, there is a constant $c$ such that

$$
\int_{F}\left|c \phi-p^{*} \psi\right| \leqq O\left(i(F)^{\alpha}\right)
$$

for some fixed exponent $\alpha$.

Proof. Choose a coordinate $z$ so that $F$ can be identified with the unit disk $\Delta$. After an appropriate rotation, $p^{*} \psi=r^{2} d z^{2}$ so $p^{*} \mu=d \bar{z} / d z$. By Theorem 5.1 on the flex of the sphere in $Q(\Delta)$,

$$
\int_{F}\left|\frac{\phi}{m(F)}-\frac{d z^{2}}{\pi}\right| \leqq O\left(i(F)^{\alpha}\right)
$$

and multiplying through by $\pi r^{2}$ we obtain the Lemma.

Corollary 7.2. For all intersecting $F$ and $F^{\prime}$ in $\mathscr{F}$, and for all $i_{0}$ sufficiently small (independent of $\phi$ ),

1. If the inefficiency $i(F)$ is less than $i_{0}$, then $m\left(F^{\prime}\right) \geqq O(m(F))$;

2. If $i(F)$ and $i\left(F^{\prime}\right)$ are both less than $i_{0}$, then $m\left(F^{\prime}\right) \geqq \lambda m(F)$, where $\lambda=1-O\left(i_{0}^{\alpha}\right)$.

In other words, efficient neighbors have nearly the same mass, and a definite amount of mass is inherited from an efficient neighbor. 
Proof. Assume both inefficiencies are near 0. By the Lemma above, there are constants $c$ and $c^{\prime}$ such that $c \phi$ and $c^{\prime} \phi$ are $L^{1}$-close to $p^{*} \psi$ on $F$ and $F^{\prime}$ respectively. Thus $m(F)$ and $m\left(F^{\prime}\right)$ are given approximately by $\pi r^{2} / c$ and $\pi r^{2} / c^{\prime}$.

Now $F$ and $F^{\prime}$ overlap a definite amount with respect to $p^{*} \psi$-area, since their overlap is bounded below by the minimum overlap in $\mathscr{E}$. It follows that the constants $c$ and $c^{\prime}$ must be close, else there would be a definite deviation from $p^{*} \psi$ on the overlap. Making this precise yields the bound given.

If only $i(F)$ is near zero, it still follows that the $\phi$-mass of the overlap is a definite fraction of $m(F)$. The overlapping mass also contributes to $m\left(F^{\prime}\right)$, so $m\left(F^{\prime}\right) \geqq O(m(F))$.

Lemma 7.3. For any subset $\mathscr{F}^{\prime}$ of $\mathscr{F}$,

$$
m\left(\bigcup \mathscr{F}^{\prime}\right) \geqq \frac{1}{|\mathscr{E}|} \sum_{\mathscr{F}} m(F)
$$

and

$$
i\left(\bigcup \mathscr{F}^{\prime}\right) \geqq \frac{\inf \left\{i(F): F \in \mathscr{F}^{\prime}\right\}}{|\mathscr{E}|}
$$

Proof. The degree of the Čech graph of $\mathscr{F}$ is at most $|\mathscr{E}|-1$, so Proposition 2.2 applies to give an independent subset $\mathscr{F}^{\prime \prime} \subset \mathscr{F}^{\prime}$ which carries at least $1 /|\mathscr{E}|$ the sum of $m(\cdot)$ over $\mathscr{F}^{\prime}$. Then $\bigcup \mathscr{F}^{\prime \prime}$ is a disjoint union (this is what independence means), so the mass is additive, establishing the first inequality.

By disjointness, $i\left(\bigcup \mathscr{F}^{\prime \prime}\right)$ is bounded below by the minimum inefficiency of members of $\mathscr{F}^{\prime}$. Estimating $i\left(\bigcup \mathscr{F}^{\prime}\right)$ by the weighted average of the inefficiency of $\bigcup \mathscr{F}$ " and its complement gives the second inequality.

Proof of Theorem 6.1 ( $\Theta$ contracts fibers). Let $\gamma>0$ denote the expansion of the (nonamenable) Čech graph of $\mathscr{F}$. Choose $\lambda<1$ such that $\lambda \gamma+\lambda-1 \geqq \gamma / 2$. Let

$$
\mathscr{V}=\left\{F \in \mathscr{F}: i(F)<i_{0}\right\},
$$

where $i_{\mathrm{o}}>0$ is chosen sufficiently small that we may apply Corollary 7.2 to conclude:

(I) $m\left(F^{\prime}\right) \geqq O(m(F))$ whenever $F^{\prime}$ is adjacent to an element $F \in \mathscr{V}$; and

(II) $m\left(F^{\prime}\right) \geqq \lambda m(F)$ whenever $F$ and $F^{\prime}$ are adjacent elements of $\mathscr{V}$.

Let

$$
\mathscr{W}=\left\{F \in \mathscr{F}: i(F) \geqq i_{0}\right\} .
$$

Then $(\mathscr{V}, \mathscr{W})$ forms a partition of $\mathscr{F}$. Let $W=\bigcup \mathscr{W}, V=\bigcup \mathscr{V}$.

By Lemma $7.3, i(W) \geqq i_{0} /|\mathscr{E}|$, so

$$
1-\|\Theta(\phi)\|=i(Y) \geqq m(W) i(W) \geqq i_{0} m(W) /|\mathscr{E}|
$$

and hence to bound $\|\Theta(\phi)\|$ away from 1, it suffices to bound $m(W)$ from below. 
Assume $\|\Theta(\phi)\| \geqq 1 / 2$, since otherwise we are done. Then

$$
m(W)+m(V)=\int_{p^{-1}\left(\bigcup^{\mathscr{E}}\right)}|\phi| \geqq\|\Theta(\phi)\| \int_{\bigcup^{\mathscr{E}}}|\psi|=O(1)
$$

so to complete the proof it suffices to show the mass of $W$ is not too much smaller than the mass of $V$.

For $F$ in the border $b(\mathscr{V}) \subset \mathscr{W}$, define

$$
M(F)=\sup \left\{m\left(F^{\prime}\right): F^{\prime} \in \mathscr{V} \text { and } F^{\prime} \text { is adjacent to } F .\right\}
$$

By (I), $m(F) \geqq O(M(F))$, so by Lemma 7.3 the mass of $W$ is estimated from below in terms of the sum of $M(F)$ over the border of $\mathscr{V}$. We will show this sum is comparable to the mass of $V$.

By (II), $m(\cdot)$ is a slowly varying function on the vertices $\mathscr{V}$ of the Čech graph; applying Theorem 2.1 ,

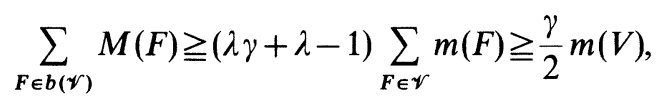

establishing the theorem.

\section{Completion of the nonamenable case}

It is known that the operator $\Theta$ is always surjective; this fundamental property is called completeness of Poincaré series. By the open mapping theorem, the image of the unit ball in $Q(Y)$ contains a neighborhood of the origin in $Q(X)$. This can be made effective:

Theorem 8.1. Let $Y \rightarrow X$ be a covering of a hyperbolic Riemann surface $X$. Then

$$
\Theta\left(B_{Y}\right) \supset \frac{1}{3} B_{X}
$$

i.e. the image of the unit ball in $Q(Y)$ contains the ball of radius $1 / 3$ in $Q(X)$.

This result is due to Ahlfors and Bers; for a proof, see [Kra 1, Theorem 3.3], or [Gar, § 4.3].

Proof of Theorem 1.1 (nonamenable case). Let $Y \rightarrow X$ be a nonamenable covering of a hyperbolic Riemann surface $X$. Suppose $\psi$ is an element of norm 1 in $\overline{\Theta\left(B_{Y}\right)}$. Then there is a sequence $\phi_{n}$ in $B_{Y}$ such that $\Theta\left(\phi_{n}\right) \rightarrow \psi$. By the Theorem above, there exist $\phi_{n}^{\prime} \rightarrow 0$ with $\Theta\left(\phi_{n}^{\prime}\right)=\psi-\Theta\left(\phi_{n}\right)$. Then $\Theta\left(\phi_{n}+\phi_{n}^{\prime}\right)=\psi$ and $\left\|\phi_{n}+\phi_{n}^{\prime}\right\| \rightarrow 1$, contradicting Theorem 6.1 ( $\Theta$ contracts fibers).

Therefore the closure of $\Theta\left(B_{Y}\right)$ is contained in the interior of the unit ball in $Q(X)$. 


\section{The amenable case}

Let $p: Y \rightarrow X$ be an amenable covering of a hyperbolic Riemann surface $X$.

Identifying the unit disk $\Delta$ with the universal cover of $X$, we may realize the fundamental groups of $X$ and $Y$ as Fuchsian groups $\Gamma_{Y} \subset \Gamma_{X}$ acting on the disk.

By amenability, there exist finite sets of cosets $\mathscr{C}_{n} \subset \Gamma_{X} / \Gamma_{Y}$ such that for any finite set $F \subset \Gamma_{X}$,

$$
\frac{\left|\left\{C: F \cdot C \subset \mathscr{C}_{n}\right\}\right|}{\left|\mathscr{C}_{n}\right|} \rightarrow 1
$$

as $n \rightarrow \infty$. Here $F \cdot \mathscr{C}$ denotes the set of cosets obtained by translating $C$ on the left by elements of $F$.

It will be useful to consider the $\Theta$ operator on the space of measurable integrable quadratic differentials, which we denote by $L^{1}(\cdot)$, and which contains $Q(\cdot)$ as a closed subspace. Corresponding to the factorization of the universal covering $u: \Delta \rightarrow X$ through $Y$, we have a factorization of

$$
\Theta_{\Delta / X}: L^{1}(\Delta) \rightarrow L^{1}(X)
$$

as $\Theta_{\Delta / X}=\Theta_{Y / X} \circ \Theta_{\Delta / Y}$.

For each $\mathscr{C}_{n}$, define an averaging operator $A_{n}: L^{1}(\Delta) \rightarrow L^{1}(Y)$ as follows:

$$
A_{n}(\xi)=\frac{1}{\left|\mathscr{C}_{n}\right|} \sum_{\left\{g \Gamma_{Y}\right\} \in \mathscr{C}_{n}} \Theta_{\Delta / \mathbf{Y}}\left(g^{*} \xi\right) .
$$

Since $\Theta_{\Delta / Y}\left(h^{*} g^{*} \xi\right)=\Theta_{\Delta / Y}\left(g^{*} \xi\right)$ whenever $h$ is in $\Gamma_{Y}$, this expression does not depend on what $g$ we choose to represent a given coset.

The following properties are easily verified:

- $\left\|A_{n}\right\| \leqq 1$.

- $A_{n}$ carries $Q(\Delta)$ into $Q(Y)$.

- $\Theta_{Y / X}\left(A_{n}(\xi)\right)=\Theta_{\Delta / X}(\xi)$.

Theorem 9.1. As $n \rightarrow \infty$,

$$
\left\|A_{n}(\xi)\right\| \rightarrow\left\|\Theta_{\Delta / X}(\xi)\right\|
$$

Proof. Let $\Omega \subset \Delta$ denote a measurable fundamental domain for the action of $\Gamma_{X}$ on the disk. We may assume $\xi$ is an integrable function whose support is contained in $F^{*} \Omega$, the inverse images of $\Omega$ under a finite set $F \subset \Gamma_{X}$, since such $\xi$ are dense in $L^{1}(\Delta)$.

The function $\psi=\Theta_{\Delta / X}(\xi)$ is obtained by collecting together the finitely many tiles which support $\xi$, superimposing them and adding up the corresponding values of $\xi$.

The tiling of the disk by translates of $\Omega$ descends to a tiling of $Y$, such that the pre-image of a tile on $Y$ is $C^{*} \Omega$ for some coset $C$ of $\Gamma_{X} / \Gamma_{Y}$. If $F^{-1} \cdot C \subset \mathscr{C}_{n}$, call the corresponding tile of $Y$ a filled tile. 
On a filled tile,

$$
A_{n}(\xi)=p^{*}(\psi) /\left|\mathscr{C}_{n}\right|
$$

since the averaging operation moves every tile of the support of $\xi$ into $C^{*} \Omega$. The integral of $\left|p^{*} \psi\right|$ over a single tile of $Y$ is the same as the integral of $|\psi|$ over $X$, so as $n \rightarrow \infty$,

$$
\int_{Y^{\prime}}\left|A_{n}(\xi)\right|=\frac{\left|\left\{C: F^{-1} \cdot C \subset \mathscr{C}_{n}\right\}\right|}{\left|\mathscr{C}_{n}\right|}\|\psi\| \rightarrow\|\psi\|,
$$

where $Y^{\prime}$ denotes the union of the filled tiles of $Y$.

A coset $C$ corresponds to an unfilled tile in the support of $A_{n}(\xi)$ exactly when $F^{-1} \cdot C$ meets, but is not contained in, $\mathscr{C}_{n}$. Thus the number of such tiles is at most

$$
|F| \times\left|\left\{C: F^{-1} \cdot C \mp \mathscr{C}_{n}\right\}\right|
$$

which is small compared to $\left|\mathscr{C}_{n}\right|$ as $n \rightarrow \infty$. Over an unfilled tile the integral of $\left|A_{n}(\xi)\right|$ is bounded by $\|\xi\| /\left|\mathscr{C}_{n}\right|$, so the contributions to $\left\|A_{n}(\xi)\right\|$ coming from the unfilled tiles of $Y$ tend to 0, establishing the theorem.

As a corollary, we may complete the:

Proof of Theorem 1.1 (amenable case). Let $\psi$ be an element of norm $<1$ in $Q(X)$. By completeness of Poincaré series, there exists a $\xi$ in $Q(\Delta)$ such that $\Theta_{\Delta / X}(\xi)=\psi$. Setting $\phi_{n}=A_{n}(\xi)$, we have $\Theta_{Y / X}\left(\phi_{n}\right)=\psi$ and by the theorem above, $\left\|\phi_{n}\right\|$ is also eventually less than 1 . Therefore the image of the open unit ball $B_{Y}$ contains the open unit ball $B_{X}$ (and equality holds because $\left\|\Theta_{Y / X}\right\| \leqq 1$ ).

\section{Dependence on moduli}

In this section we use a result of Reich and Strebel, and the explicit expansion constants obtained in $\S 4$, to study the behavior of $\|\Theta\|$ as a function of the conformal structures on $X$ and $Y$.

Fix a covering of Riemann surfaces $Y_{0} \rightarrow X_{0}$. For any $X \in \operatorname{Teich}\left(X_{0}\right)$, there is a corresponding covering $Y \rightarrow X$.

Theorem 10.1. $\left\|\Theta_{Y / X}\right\|$ is uniformly continuous with respect to the Teichmüller metric on Teich $\left(X_{0}\right)$.

Assuming this for the moment, we specialize to the case where $X$ is of finite type. Fix a finite generating set $\mathscr{G}$ for $\pi_{1}(X)$. As remarked in $\S 6$, the covering $Y$ enters only combinatorially into the estimate for $\|\Theta\|$ :

Theorem 10.2 (Combinatorial bound). For any nonamenable covering $Y \rightarrow X$,

$$
\|\Theta\|<f(\gamma)<1
$$

where $\gamma$ is the expansion of the coset graph of $\pi_{1}(X) / \pi_{1}(Y)$ with respect to $\mathscr{G}$, and $f$ is a function which depends only on $X$. 
Proof. We simply analyze the dependence of the proof upon $Y$. Since $\pi_{1}(X)$ is finitely generated we may choose the net $\mathscr{E}$ so that $\bigcup \mathscr{E}$ carries the fundamental group of $X$. The expansion of $p^{*} \mathscr{E}$ is then bounded below in terms of the expansion of the coset graph, and so the corollary is true fiberwise. Using Theorem 8.1, one sees that a bound $\left\|\Theta \mid Q_{\psi}(Y)\right\|<1-c<1$ provides a bound of $1-c / 2$ for all nearby $\psi$, and the corollary follows globally by compactness of $\overline{B_{X}}$.

Remark. A more careful analysis shows we may take $f(\gamma)=1-O\left(\gamma^{\beta}\right)$ for some exponent $\beta$.

Now suppose $\pi_{1}\left(Y_{0}\right)$ is finitely generated, $X_{0}$ is a hyperbolic Riemann surface of genus $g$ with $n$ punctures, and $Y_{0} \rightarrow X_{0}$ is an infinite-sheeted covering. Let $\mathscr{M}_{g, n}$ denote the moduli space of $X_{0}$, and define

by

$$
N: \mathscr{M}_{\mathrm{g}, n} \rightarrow[0,1]
$$

$$
N(Z)=\sup \left\{\left\|\Theta_{Y / X}\right\|: X \in \operatorname{Teich}(X) \text {, and }[X]=Z\right\},
$$

where $[X]$ denotes the location of $X$ in moduli space.

Theorem 10.3. $N$ is a uniformly continuous proper map from moduli space to the half-open interval $[0,1)$. Moreover, on any compact subset of $\mathscr{M}_{\mathrm{g}, n}, N$ is bounded away from 1 by an amount which depends only on the number of generators of $\pi_{1}(Y)$.

\section{Remarks.}

1. Properness means $\|\Theta\| \rightarrow 1$ as $Z$ tends to infinity in moduli space. Thus there is no uniform bound over all $\mathscr{M}_{g, n}$.

2. Theorem 1.4 is an immediate corollary.

Proof. By Theorem 10.1, $N$ is uniformly continuous in the Teichmüller metric on moduli space. The combinatorial bound of Theorem 10.2, combined with the lower bound for the expansion factor $\gamma$ obtained in Corollary 4.2, bounds $N$ away from 1 by an amount which depends only on the Euler characteristic of $Y$, not its position over $X$. Thus $N$ maps moduli space into $[0,1)$, and on any compact set there is a bound which depends only on the topological complexity of $Y$.

To see $N$ is proper, we must show $\|\Theta\| \rightarrow 1$ as $[X] \rightarrow \infty$ in moduli space. One knows in the end of moduli space the length $L$ of some simple geodesic $\sigma$ on $X$ is near zero. We will show $N([X])$ is within $O(L)$ of 1 .

Let $W \rightarrow X$ be the covering space for a cyclic subgroup generated by $\sigma$. $W$ may be identified with the annulus:

$$
\left\{z: 1<|z|<\exp \left(2 \pi^{2} / L\right)\right\} .
$$

Most of the mass of the quadratic differential $\phi=d z^{2} / z^{2}$ is concentrated in the thin part of $W$, which injects into the thin part of $X$. The remainder causes only a small amount of cancellation; one may estimate $\left\|\Theta_{W / X}\right\|>1-O(L)$. Since $\Delta \rightarrow W$ is an amenable covering, $\Theta\left(B_{\Delta}\right)=B_{W}$ by Theorem 1.1 and so

$$
\left\|\Theta_{\Delta / X}\right\|=\left\|\Theta_{W / X}\right\|>1-O(L) .
$$

On the other hand $\Theta_{\Delta / X}$ factors through $\Theta_{Y / X}$, so the same lower bound holds for $N$. 
Proof of Theorem 10.1. It does not appear that continuity of the norm is a formal consequence of its definition; to obtain continuity we appeal to a geometric interpretation.

Denote by $p$ the induced map Teich $\left(X_{0}\right) \rightarrow$ Teich $\left(Y_{0}\right)$. Letting $Y$ and $Y^{\prime}$ denote $p(X)$ and $p\left(X^{\prime}\right)$ respectively, set

$$
N(X, r)=\frac{\sup \left\{d\left(Y, Y^{\prime}\right): d\left(X, X^{\prime}\right)<r\right\}}{r} .
$$

$N(X, r)$ is the factor by which $p$ compresses the Teichmüller radius of the ball of radius $r$ at $X$.

It is known that $N(X, r) \rightarrow\left\|\Theta_{Y / X}\right\|$; in fact, the rate of convergence is uniform over all Teichmüller spaces.

More precisely, let $f: X \rightarrow X^{\prime}$ be a quasiconformal map, $\mu$ the complex dilatation of its lift $f: Y \rightarrow Y^{\prime}$, and $v$ the dilatation of an extremal map $g: Y \rightarrow Y^{\prime}$ equivalent to $\tilde{f}$. By a result of Reich and Strebel ([RS, Theorem 9], cf. [Gar, $\S 6.4])$ :

$$
\left|\frac{\|v\|_{\infty}}{1-\|v\|_{\infty}^{2}}-\sup _{\phi \in B_{X_{0}}} \operatorname{Re} \int \frac{\mu \phi}{1-\|\mu\|_{\infty}^{2}}\right| \leqq \frac{\|\mu\|_{\infty}^{2}}{1-\|\mu\|_{\infty}^{2}} .
$$

This implies

$$
\left|N(X, r)-\left\|\Theta_{Y / X}\right\|\right|<C r
$$

for a universal constant $C$.

Since $p$ is distance non-increasing,

$$
\left|N(X, r)-N\left(X^{\prime}, r\right)\right| \leqq 2 s / r
$$

where $s$ is the Teichmüller distance from $X$ to $X^{\prime}$. Setting $r=\sqrt{s}$ and combining these inequalities, we find $\left\|\Theta_{Y / X}\right\|$ and $\left\|\Theta_{Y^{\prime} / X^{\prime}}\right\|$ differ by at most $C^{\prime} \sqrt{s}$.

Remark. The proof gives uniform Hölder continuity of exponent $1 / 2$.

\section{Refinements}

To get a more precise picture of the way in which the norm of $\Theta$ can tend to 1 , in this section we study the fiberwise behavior.

Definitions. Let $p: Y_{0} \rightarrow X_{0}$ denote an infinite-sheeted covering with $\pi_{1}\left(Y_{0}\right)$ finitely generated and $X_{0}$ of finite type.

Assume $Y_{0}$ is a geometric covering; i.e. there is a proper incompressible subsurface $S_{0} \subset X_{0}$ such that $Y_{0}$ is the covering space for the subgroup $\pi_{1}\left(S_{0}\right)$.

Since surface groups are LERF [Scott], a general finitely generated covering becomes geometric after passing to a finite covering of $X_{0}$. Finite coverings induce isometries on Teichmüller space, so the study of contraction properties of Teich $\left(X_{0}\right) \rightarrow$ Teich $\left(Y_{0}\right)$ can in principle be reduced to the case of geometric coverings. We restrict attention to the geometric case to get a clearly formulated result. 
Fix any small $\varepsilon>0$. The thin part $X_{\text {thin }}$ of $X \in \operatorname{Teich}\left(X_{0}\right)$ is the subset where the injectivity radius in the Poincare metric is less than $\varepsilon$. For $\varepsilon$ sufficiently small every component of $X_{\text {thin }}$ has abelian (hence amenable) fundamental group.

For any $X \in \operatorname{Teich}\left(X_{0}\right)$, there is subsurface $S$ corresponding to $S_{0}$. The liftable part $X_{\text {lift }}$ of $X$ is the union of those components of $X_{\text {thin }}$ and $X-X_{\text {thin }}$ which are isotopic into $S$. $X_{\text {thin }} \cup X_{\text {lift }}$ is the amenable part $X_{\mathrm{am}}$ of $X$.

By definition of a geometric cover, $S$ lifts isomorphically to a unique subsurface of $Y$. Thus $X_{\text {lift }}$ also has a unique isomorphic lift to $Y$. The amenable part $Y_{\text {am }}$ of $Y$ is the union of this lift and the total pre-image of $X_{\text {thin }}$.

Intuitively, $Y_{\mathrm{am}}$ is the part of $Y$ which is converging to an amenable cover. Our next results asserts that the only way $\|\Theta\|$ can tend to 1 is by taking advantage of the part of $Y$ which is becoming more amenable.

Theorem 11.1. Let $\phi \in Q(Y),\|\phi\|=1$. If $\left\|\Theta_{Y / X}(\phi)\right\|$ is close to 1 , then most of the mass of $|\phi|$ is in the amenable part of $Y$.

More precisely, if unit norm $\phi_{n}$ on $Y_{n} \rightarrow X_{n}$ satisfy $\left\|\Theta_{Y_{n} / X_{n}}\left(\phi_{n}\right)\right\| \rightarrow 1$, then

$$
\int_{\left(Y_{n}\right)_{\mathrm{am}}}\left|\phi_{n}\right| \rightarrow 1
$$

as $n \rightarrow \infty$.

For each $X \in \operatorname{Teich}\left(X_{0}\right)$ and nonzero $\psi \in Q(X)$, define

$$
N(X, \psi)=\left\|\Theta_{Y / X} \mid Q_{\psi}(X)\right\|,
$$

i.e. $N(X, \psi)$ is the fiberwise norm of $\Theta$ over $\psi$. The fiberwise norm only depends on the location of $\psi$ in the compact projective space $\mathbf{P} Q(X)$.

Corollary 11.2. If $N(X, \psi)$ is near 1 , then most of the mass of $|\psi|$ is in the amenable part of $X$.

Proof. The amenable part of $Y$ lies over the amenable part of $X$.

Corollary 11.3. If $N(X, \psi)$ is near 1 for the universal covering, then most of the mass of $|\psi|$ is in the thin part of $X$.

Proof. In this case $X_{\text {am }}=X_{\text {thin }}$.

Proof of the Theorem 11.1. We will use the idea of geometric limits of quadratic differentials and their underlying Riemann surfaces, developed in the Appendix below.

Let $\psi_{n}=\Theta_{Y_{n} / X_{n}}\left(\phi_{n}\right)$ where $\left\|\phi_{n}\right\|=1$, and assume $\left\|\psi_{n}\right\| \rightarrow 1$.

The portion of $\left|\phi_{n}\right|$ which lies over the thin part of $X_{n}$ is already carried by the amenable part of $Y_{n}$, by definition. Any other portion lies over a component of the thick part of $X_{n}$.

Let $Z_{n}$ be any component of the thick part of $X_{n}$, and fix $\delta>0$. We will show that the limsup of the $\left|\phi_{n}\right|$-mass of $p^{-1}\left(Z_{n}\right)-\left(Y_{n}\right)_{\text {am }}$ is at most $\delta$. Since $\delta$ is arbitrary and the number of thick components is bounded, this will complete the proof: the amenable part carries most of the mass of $\left|\phi_{n}\right|$ when $n$ is large. 
Since $\phi_{n}$ is pushing down more and more efficiently,

for any $E \subset X_{n}$. If

$$
\left|\int_{E}\right| \psi_{n}\left|-\int_{p^{-1}(E)}\right| \phi_{n}\|\leqq 1-\| \psi_{n} \| \rightarrow 0
$$

$$
\int_{z_{n}}\left|\psi_{n}\right| \rightarrow 0
$$

then the $\left|\phi_{n}\right|$-mass of its entire pre-image tends to zero and there is nothing to prove.

So assume the $\left|\psi_{n}\right|$-mass of $Z_{n}$ is bounded below by $m$. Choose a baseframe $v_{n}$ over any point of $Z_{n}$. By compactness of the space $\mathbf{P} \mathscr{Q}_{g, n}$ of projective classes of quadratic differentials viewed at the scale of the injectivity radius (Theorem A 3.1 below), we may pass to a subsequence such that $\left(X_{n}, v_{n}, c_{n} \psi_{n}\right)$ converges geometrically to $(X, v, \psi)$. Here $c_{n}$ is a sequence of nonzero constants and $\psi \neq 0$. Since the $\left|\psi_{n}\right|$-mass of $Z_{n}$ is between $m$ and 1 , we may assume $c_{n}=1$.

The limiting Riemann surface $X$ is hyperbolic and of finite type, since the baseframe was chosen in the thick part. $Z_{n}$ converges geometrically to a component $Z$ of the thick part of $X$.

Construct a finite net $\mathscr{E}$ on $Z$ composed of embedded $r$-balls in the $|\psi|$-metric, as in $\S 6$, such that $\mathscr{E}$ carries the fundamental group of $Z$ and the $|\psi|$-mass of $\bigcup \mathscr{E}$ is within $\delta$ of that of $Z$. By taking $r$-balls in the $\left|\psi_{n}\right|$-metric about points converging geometrically to the centers of the balls in $\mathscr{E}$, we can construct nets $\mathscr{E}_{n}$ converging geometrically to $\mathscr{E}$ for all $n$ sufficiently large. Then the geometric data concerning $\mathscr{E}_{n}$ (enumerated in $\S 6$ ) on which the bound obtained in Theorem 6.1 ( $\Theta$ contracts fibers) depends, converges to the data for $\mathscr{E}$.

Thus, if $\lim \left\|\psi_{n}\right\|=1$, the combinatorial input to the fiberwise bound - the expansion $\gamma_{n}$ of the Čech graph of $p^{*} \mathscr{E}_{n}-$ must tend to zero.

$Z_{n}$ must belong to the liftable part of $X$ for all large $n$; otherwise the Čech graph corresponds to a covering by a proper incompressible subsurface of $Z_{n}$, whose expansion is bounded below by Corollary 4.2 (Uniform expansion).

So assume that $Z_{n}$ is isotopic into the subsurface of $X_{n}$ defining the covering $Y_{n}$. Then the Čech graph of $p^{*} \mathscr{E}_{n}$ consists of countably many components, one of which is finite (and corresponds to the isomorphic lift of $Z_{n}$ to $\left.\left(Y_{n}\right)_{a m}\right)$, and the rest of which are infinite (and correspond to the universal cover of $Z_{n}$ ). The expansion $\gamma$ is uniformly bounded below on the infinite components; thus the $\left|\phi_{n}\right|$-mass of $p^{-1}\left(\bigcup \mathscr{E}_{n}\right)-\left(Y_{n}\right)_{\text {am }}$ tends to zero. Since the $\left|\psi_{n}\right|$-mass of $Z_{n}-\bigcup \mathscr{E}_{n}$ is at most $\delta$, the limsup of the $\left|\phi_{n}\right|$-mass of $p^{-1}\left(Z_{n}\right)-\left(Y_{n}\right)_{\text {am }}$ is also at most $\delta$, as claimed.

\section{Quotients of bounded domains}

Poincaré series on the disk have a natural generalization in the setting of automorphic forms of weight $q \geqq 2$ for the action of a discrete group $\Gamma$ on a bounded domain $\Omega \subset \mathbf{C}^{n}$ (see e.g. [Ba]). The quotient space $X=\Omega / \Gamma$ has the structure of a normal complex analytic space, and automorphic forms of weight $d$ correspond to holomorphic sections of the $q$ th power of the canonical bundle of $X$; call such a section a $q$-canonical form. 
Let $K(z, w) d z d \bar{w}$ denote the Bergman kernel on $\Omega$, where $d z=d z_{1} \ldots d z_{n}$ as usual. Then $|K(z, z)||d z|^{2}$, the Bergman density is a natural volume form on $\Omega$, invariant under any holomorphic automorphism. Using this volume element, one may define Banach spaces $H_{q}^{p}(\Omega)$ consisting of holomorphic $q$-canonical forms $\phi(z) d z^{q}$ such that

$$
\|\phi\|=\int_{\Omega}|\phi(z)| \cdot|K(z, z)|^{1-q / 2}|d z|^{2}<\infty .
$$

One may define Banach spaces $H_{q}^{p}(X)$ in a similar fashion, since the Bergman density descends to quotients. These Banach spaces of automorphic forms have been considered by Earle and others [Earle].

If $Y$ is a quotient intervening between $X$ and $\Omega$, there is a Poincare series operator

$$
\Theta_{q}: H_{q}^{1}(Y) \rightarrow H_{q}^{1}(X),
$$

which satisfies $\left\|\Theta_{q}\right\| \leqq 1$.

In general, the Poincaré series is not defined for elements of $H_{q}^{p}(Y)$ with $p>1$, just as the sequence $a_{n}=1 /|n+1|$ in $l^{p}(\mathbf{Z})$ (for $p>1$ ) cannot be averaged to become $\mathbf{Z}$-invariant.

Theorem 1.1 may be partly generalized to this setting, as follows:

Theorem 12.1. Let $B_{X}, B_{Y}$ denote the unit balls in $H_{q}^{1}(Y)$ and $H_{q}^{1}(X)$ respectively.

1. If $Y \rightarrow X$ is nonamenable, then $\overline{\Theta_{q}\left(B_{Y}\right)}$ is contained in the interior of $B_{X}$.

2. If $Y \rightarrow X$ is amenable and $\Omega$ is a bounded symmetric domain, then $\Theta_{q}\left(B_{Y}\right)=B_{X}$.

Sketch of the Proof. The amenable case is a straightforward generalization of the proof for quadratic differentials on the disk, once given surjectivity of $\Theta_{q}$. Surjectivity seems to be unknown in general, but it is established for quotients of bounded symmetric domains by Earle and Resnikoff, see [Earle].

For the nonamenable case, the key points are the following.

First, the proof for quadratic differentials may be modified to avoid use of completeness of Poincaré series; in fact, the demonstration that $\Theta$ uniformly contracts the space $Q_{\psi}(Y)$ works just as well so long as $\Theta(\phi)$ is close to a multiple of $\psi$.

Secondly, away from its zeros any $q$-canonical form $\phi(z) d z^{q}$ is equal to $d z^{q}$ for appropriate choice of chart.

Finally, in such a chart the ratio of the Bergman density to the volume element $|d z|^{2 n}$ varies smoothly, so it is nearly constant on sufficiently small neighborhoods. The local problem of generalizing Theorem 5.1 so it applies in these neighborhoods is fairly straightforward. The global considerations are only combinatorial and carry over immediately.

\section{Appendix. Geometric limits of quadratic differentials}

\section{A.1. Introduction}

In this appendix, we consider geometric limits for Riemann surfaces and quadratic differentials viewed at the scale of the injectivity radius. The central result 
is a compactification of the space $\mathbf{P} \mathscr{Q}_{\mathbf{g}, n}$ of projective integrable quadratic differentials on pointed Riemann surfaces of genus $g$ with $n$ punctures.

This compactification formalizes our intuition: the foliations of an integrable quadratic differential cannot swirl too much on a Riemann surface of finite volume and fixed topological type.

\section{A.2. Riemann surfaces in the geometric topology}

Definitions. For $\kappa \in[-1,1]$, consider the metric

$$
d s_{\kappa}=\frac{4|d z|}{\left(4+\kappa|z|^{2}\right)}
$$

of constant curvature $\kappa$ on a domain $U_{\kappa}$ which is the Riemann sphere if $\kappa$ positive, the complex plane $\mathbf{C}$ if $\kappa=0$ and the disk

$$
U_{\kappa}=\{z:|z|<R\}
$$

where $R=2 / \sqrt{-\kappa}$ if $\kappa<0$. In this metric, $U_{\kappa}$ is complete.

Let $\mathscr{X}$ denote the space of all pairs $\left(U_{\kappa}, \Gamma\right)$ where $\Gamma$ is a discrete group of Möbius transformations acting freely on $U_{\kappa}$. To this data we associate a Riemann surface $X=U_{\kappa} / \Gamma$ with base-frame $v \in T_{1}(X)$ by setting $v$ to be the image of a unit vector at the origin pointing along the positive real axis. We require that the injectivity radius of $X$ is $\geqq 1$ at $v$.

Conversely, a Riemann surface $(X, v)$ with a complete metric of constant curvature $\kappa \in[-1,1]$ and base-frame $v$ over a point of injectivity radius $\geqq 1$ uniquely determines an element of $\mathscr{X}$.

Every Riemann surface $X$ admits a complete metric of constant curvature, and so has many representatives in $\mathscr{X}$, corresponding to scaling the metric so its injectivity radius at a basepoint becomes at least 1 . If $X$ is hyperbolic, we will refer to its complete metric of constant curvature -1 as the Poincaré metric on $X$.

We give $\mathscr{X}$ the geometric topology: a sequence of pairs converges iff the curvatures $\kappa$ converge and the groups $\Gamma$ converge in the Hausdorff topology on closed subsets of $\mathrm{PSL}_{2} \mathrm{C}$.

Proposition A.2.1. The space of Riemann surfaces $\mathscr{X}$ is compact in the geometric topology.

Proof. The space of closed subgroups of a Lie group such as $\mathrm{PSL}_{2} \mathrm{C}$ is compact in the Hausdorff topology. The limiting groups are discrete by our condition on the injectivity radius.

This proposition is well-known and goes back to Chabauty, who introduced the geometric topology in the general setting of a locally compact topological group [Cha]; see also [Har], [Th 2].

Geometric limits of Riemannian manifolds with controlled curvature have been considered much more generally; see [Grom]. The geometric topology 
coincides with the quasiisometric topology, in which a neighborhood of $(X, v)$ consists of those $(Y, w)$ admitting a smooth near-isometry taking $v$ to $w$ defined on a large compact subset of $X$. Nearby surfaces need not be homeomorphic.

Example. Let $X$ be a hyperbolic Riemann surface with a puncture, $v_{n}$ a sequence of base-frames tending to the omitted point. Rescale the Poincaré metric so the injectivity radius at $v_{n}$ remains greater than one. The limiting Riemann surface is an infinite cylinder - or a plane, if we rescale so the injectivity radius tends to infinity.

Our main concern will be with holomorphic data on $X$, which is independent of the metric. Rather, the metric provides a convenient way to pass smoothly from hyperbolic to parabolic Riemann surfaces, as in the example.

\section{A.2.1. Compact surfaces with punctures}

A Riemann surface $X$ is of finite type if it is obtained from a compact surface by removing a finite set of points; these points are the punctures of $X$. Let $\mathscr{X}_{g, n} \subset \mathscr{X}$ denote the space of finite type Riemann surfaces of genus $g$ with $n$ punctures.

We can compactify $\mathscr{X}_{\mathbf{g}, \boldsymbol{n}}$ by forming its closure in $\mathscr{X}$.

Proposition A.2.2. For $n>0$,

$$
\overline{\mathscr{X}_{g, n}}=\bigcup\left\{\mathscr{X}_{h, m}: 2 h+m \leqq 2 g+n, 0 \leqq h \leqq g, 1 \leqq m\right\} .
$$

while for $n=0$,

if $g>0$; and

$$
\overline{X_{g, 0}}=\mathscr{X}_{\mathrm{g}, 0} \cup \overline{\mathscr{X}_{\mathrm{g}-1,2}},
$$

$$
\overline{\mathscr{X}_{0,0}}=\mathscr{X}_{0,0} \cup \mathscr{X}_{0,1} .
$$

In particular, the space of hyperbolic surfaces of genus $g$ with $n$ punctures is compactified by the plane, the punctured plane and hyperbolic surfaces of smaller complexity.

Sketch of the proof. Genus and volume can only decrease in the geometric limit. When combined with Gauss-Bonnet and the fact that a limit of noncompact surfaces is noncompact, this implies the closure of $\mathscr{X}_{g, n}$ is no larger than stated. The reverse inclusion is established by surgery: joining surfaces at punctures, or gluing pairs of punctures together; and by obvious scaling limits, such as a sequence of larger and larger spheres converging to the plane.

\section{A.2.2. The universal curve}

Over $\mathscr{X}$ one can construct the universal curve $\mathscr{C}$, the space whose fiber over $(X, v)$ is $X$ itself. To define $\mathscr{C}$, start with the bundle of universal covers

$$
\left\{\left(z,\left(U_{\kappa}, \Gamma\right)\right): z \in U_{\kappa}\right\} \subset \hat{\mathbf{C}} \times \mathscr{X} \rightarrow \mathscr{X}
$$


and form the quotient space $\mathscr{C}$ by collapsing the fiber over $\left(U_{\kappa}, \Gamma\right)$ by the action of $\Gamma$. We give $\mathscr{C}$ the quotient topology; the quotient map is a local homeomorphism.

This construction provides a way to formalize many notions whose intuitive meaning is clear.

For example, we say a closed set $E \subset X$ is a geometric limit of $E_{n} \subset X_{n}$ if $E_{n} \rightarrow E$ in the Hausdorff topology on closed subsets of the universal curve. The convergence is faithful if any neighborhood of $E$ contains $E_{n}$ for all $n$ sufficiently large; this means no points are lost in the limit by straying to infinity.

Similarly, say a sequence of continuous maps $f_{n}: X_{n} \rightarrow Z$ converges to $f: X \rightarrow Z$ in the geometric topology if their graphs converge in the Hausdorff topology on $\mathscr{C} \times Z$. This definition will be useful in describing the geometric topology on quadratic differentials and rational maps.

\section{A.2.3. Quadratic differentials}

Let $\mathscr{Z} \rightarrow \mathscr{X}$ denote the space of triples $(X, v, \phi)$ where $\phi(z) d z^{2}$ is a holomorphic quadratic differential on $X$. The canonical bundles on each $(X, v)$ in $\mathscr{X}$ piece together to form a continuous complex line bundle $K \rightarrow \mathscr{C}$ on the universal curve, with holomorphic structure along each fiber. The topology on $\mathscr{Q}$ is simply the geometric topology on continuous maps whose target $Z$ is total space of the bundle $K \otimes K$.

In down-to-earth terms, a sequence of quadratic differentials converges if the underlying Riemann surfaces converge and lifts of the differentials to the universal covers converge uniformly on compacta.

Note that we impose no growth constraints on $\phi$ and allow essential singularities; however those $\phi$ arising in the compactification of $\mathbf{P} \mathscr{Q}_{g, n}$ will have only finite order poles at punctures.

\section{A.2.4. Covering spaces}

Let $\mathscr{Y} \subset \mathscr{X} \times \mathscr{X}$ denote the 'universal covering space'. An element of $\mathscr{Y}$ is given by a pair of pointed Riemann surfaces $((Y, w),(X, v))$ admitting a covering map $p: Y \rightarrow X$ whose derivative sends $w$ to $v$. The map $p$ is redundant data; if it exists, it is unique. The geometric topology on $\mathscr{Y}$ is inherited from $\mathscr{X} \times \mathscr{X}$; it's easy to check that $\mathscr{Y}$ is a closed subspace, establishing:

Proposition A.2.3. The space of coverings $\mathscr{Y}$ is compact in the geometric topology.

One may also think of $\mathscr{Y}$ as the space of triples $\left(U_{\kappa}, \Gamma_{X}, \Gamma_{Y}\right)$ where $\Gamma_{Y}$ is a subgroup of $\Gamma_{X}$.

\section{A.2.5. Poincaré series}

Let $p:(Y, w) \rightarrow(X, v)$ be a covering space, and let $\phi$ be a holomorphic quadratic differential on $Y$. The push-forward $p_{*}(\phi)$ is defined as follows: on a ball $B \subset X$, 
$p_{*}(\phi)$ is the sum of $\left(p^{-1}\right)^{*}(\phi)$ over all branches of $p^{-1}: B \rightarrow Y$. On the universal cover of $X, p_{*}$ can be expressed as a relative Poincaré series:

$$
p_{*} \phi=\sum_{[\gamma] \in \Gamma_{\mathbf{X}} / \Gamma_{\mathbf{Y}}} \gamma^{*} \phi
$$

The push-forward is a well-defined holomorphic differential so long as $\int_{p^{-1}(K)}|\phi|<\infty$ for every compact set $K$. In this case we say $\phi$ is fiberwise integrable.

How continuous is the Poincare series as a function of its input data? Consider, for example, an integrable differential $\phi$ on the universal cover of a compact hyperbolic surface $X$. For any nontrivial element $\gamma$ in the covering group, $\phi_{n}$ $=\left(\gamma^{n}\right)^{*} \phi \rightarrow 0$ geometrically, while $p_{*}\left(\phi_{n}\right)$ remains constant, so $\lim p_{*}\left(\phi_{n}\right)$ $\neq p_{*}\left(\lim \phi_{n}\right)$ in general. To obtain continuous variation of the push-forward, we must constrain the geometric topology on quadratic differentials so that significant contributions cannot disappear in the limit by straying to infinity.

To make this precise, consider a sequence of coverings $p_{n}:\left(Y_{n}, w_{n}\right) \rightarrow\left(X_{n}, v_{n}\right)$ and quadratic differentials $\phi_{n}$ on $Y_{n}$. Assume the coverings converge geometrically to $p:(Y, w) \rightarrow(X, v), \phi_{n} \rightarrow \phi$ on $Y$, and $\phi_{n}$ and $\phi$ are integrable along the fiber.

Think of $\left|\phi_{n}\right|$ as a sequence of measures on the universal curve. We say the convergence is faithful if for every $\varepsilon>0$ there is a compact set $K$ on the universal curve whose complement has $\left|\phi_{n}\right|$-measure less than $\varepsilon$ for all $n$ sufficiently large. Since there is uniform convergence on $K$ and the total mass of the remainder is small, it is easy to see:

Proposition A.2.4. The Poincaré series varies continuously under faithful convergence.

Faithful convergence is equivalent to geometric convergence coupled with the constraint that $\int_{Y_{n}}\left|\phi_{n}\right| \rightarrow \int_{Y}|\phi|$. Similarly, we may define fiberwise faithful convergence by requiring

$$
\int_{p_{n}^{-1}\left(K_{n}\right)}\left|\phi_{n}\right| \rightarrow \int_{p^{-1}(K)}|\phi|
$$

for any faithfully convergent sequence $K_{n} \rightarrow K$ of compact subsets of $X_{n} \rightarrow X$. The same reasoning justifies:

Proposition A.2.5. If $\phi_{n} \rightarrow \phi$ faithfully fiberwise, then $\left(p_{n}\right)_{*}\left(\phi_{n}\right)$ converges geometrically to $p_{*}\left(\phi_{n}\right)$.

\section{A.2.6. Systems of disjoint simple loops}

Let $\mathscr{S}$ denote the space of triples $(X, v, S)$ where $S \neq \emptyset$ is a finite system of disjoint simple closed curves on $X$ (up to isotopy), with no curve contractible and no pair isotopic. 
To define the geometric topology, first assume $X$ is hyperbolic; give $X$ its Poincaré metric. To $S$ we associate a compact subset $K(S) \subset X$ consisting of a component of the thin part for each peripheral curve or short geodesic in $S$, and a geodesic representative for the remaining elements of $S$. If $X$ is not hyperbolic, we think of all of $X$ as thin and set $K(S)=X$. Then $\left(X_{n}, v_{n}, S_{n}\right)$ converges geometrically to $(X, v, S)$ iff $K_{n}\left(S_{n}\right)$ converges faithfully to $K(S)$. (We do not allow curves to disappear in the limit by straying to infinity.)

Equivalently, $S$ may be thought of as a subset of the fundamental group $\Gamma$ of $X$ consisting of a finite union of conjugacy classes; then the geometric topology is the topology of faithful convergence of closed subsets of $\mathrm{PSL}_{2} \mathbf{C}$.

Example. Let $\left(X_{n}, v_{n}\right)$ be a sequence of punctured tori converging to a triply punctured sphere $(X, v)$ by pinching a simple geodesic $S_{n}$. Then the limiting thin part has a two components, and the limit of $S_{n}$ consists of two loops, one around each of the two new punctures. This phenomenon occurs whenever a nonseparating curve is pinched.

\section{A.2.7. Maps to $\hat{\mathbf{C}}$ of bounded degree}

Let $\mathrm{Rat}_{d}$ denote the space of rational maps $f: \hat{\mathbf{C}} \rightarrow \hat{\mathbf{C}}$ of degree $\leqq d$. Let us say $f_{n} \rightarrow f$ iff there is a finite set $E \subset \hat{\mathbf{C}}$ such that $f_{n}$ converges to $f$ uniformly on compact subsets of $\hat{\mathbf{C}}-E$. The degree of a map can decrease in the limit. With this topology, $\mathrm{Rat}_{d}$ is compact; the lower-degree maps provide a compactification for those of degree exactly $d$.

Let $\mathscr{R}_{d}$ denote the space of triples $[(X, v, f)]$, where $f: X \rightarrow \hat{\mathbf{C}}$ is a holomorphic map which is at most $d$-to-1. Motivated by the example of rational maps on the sphere, we define the geometric topology by:

$$
\left(X_{n}, v_{n}, f_{n}\right) \rightarrow(X, v, f)
$$

if the first two factors converge as before, and there exists a finite set $E \subset X$ such that $f_{n} \rightarrow f$ geometrically on compact subsets of $X-E$.

If $f$ is not the constant map to $\infty$, it is a meromorphic function; $f$ is invertible if it is also not identically 0 .

Theorem A.2.6. $\mathscr{R}_{d}$ is compact in the geometric topology. Moreover, for any sequence $f_{n}$ of invertible meromorphic functions, there exist constants $c_{n}$ such that $c_{n} f_{n}$ has a convergent subsequence whose limit is invertible.

Proof. Let $\left(X_{n}, v_{n}, f_{n}\right)$ be a sequence in $\mathscr{R}_{d}$; we may assume that the first two factors converge to a pair $(X, v)$, and each $f_{n}$ is nonconstant (otherwise the theorem is obvious).

Let $E_{n} \subset X_{n}$ denote the pre-images of 0,1 and $\infty$ under $f_{n}$. Then $\left|E_{n}\right| \leqq 3 d$. Passing to a subsequence, we may assume $E_{n}$ converges geometrically to a finite set $E \subset X$; some points of $E_{n}$ may be lost by straying far from the basepoint, but this is irrelevant. 
By Montel's theorem, $\left\langle f_{n}\right\rangle$ has a subsequence converging uniformly to a holomorphic map $f$ outside of $E$. The limiting map is at most $d$-to-1, so any singularity at $E$ is removable, and we have obtained a limiting $f: X \rightarrow \hat{\mathbf{C}}$.

The limiting map can be identically zero or infinity only if every point of $E$ is a limit of a zero or a pole of $f_{n}$. Pick a sequence $x_{n} \in X_{n}-E_{n}$ converging to $x \in X-E$, and set $c_{n}=1 / f_{n}\left(x_{n}\right)$. For the new sequence $c_{n} f_{n}(x), x$ will be a limit of the pre-images of 1 but not a limit of zeros or poles, so $f$ will be invertible.

\section{A.3. Compact spaces of quadratic differentials}

A holomorphic quadratic differential $\phi$ on a Riemann surface $X$ has a beautiful concrete picture: it is the combination of a foliation and a flat metric, with isolated singularities. One way to see the structure is to change coordinates locally so that $\phi=d z^{2}$; in these coordinates the metric is the usual Euclidean metric, and the foliation is by lines of constant imaginary part. Such a change of coordinates is always possible away from the zeros of $\phi$, and is well-defined up to post-multiplication by -1 (thus the foliation is unoriented.)

Any real multiple of $\phi$ has the same foliation. In this section we establish compactness of a class of quadratic differentials determined only up to multiples. One consequence is that the foliation of a quadratic differential on a surface of type $(g, n)$, with at most simple poles at the punctures, ranges through a compact set of pictures when viewed at the scale of the injectivity radius.
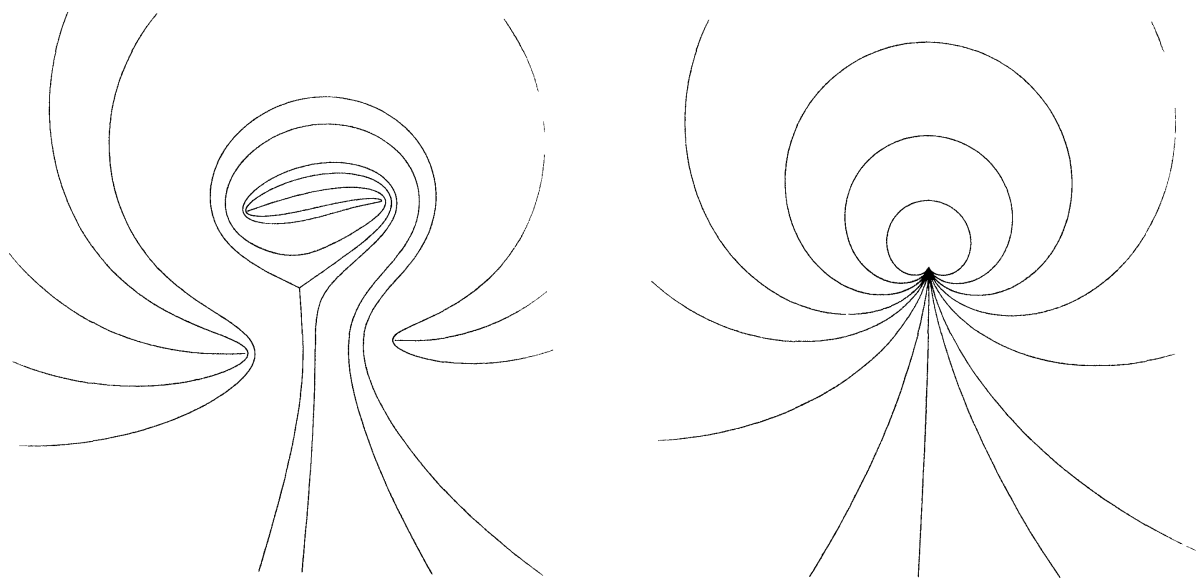

Fig. 3. The geometric limit is a triple pole

An example appears in Fig. 3. The first picture is the foliation of a quadratic differential in the plane with four simple poles (one prong singularities) and one zero (three pronged). This can be thought of as a holomorphic differential on the four-times punctured plane. Now imagine shrinking the picture towards 
the zero, while holding a basepoint a fixed distance away. The limiting Riemann surface is the once-punctured plane, and the limiting differential has a triple pole at the single puncture consolidating what was once four punctures.

Definitions. As before, 2 denotes the space of quadratic differentials with the geometric topology. Let $\mathbf{P} 2=2^{*} / \mathbf{C}^{*}$ be the quotient of the set of nonzero quadratic differentials by the multiplicative action of complex scalars, with the quotient topology.

Now restrict attention to quadratic differentials $\phi$ on Riemann surfaces in $\mathscr{X}_{g, n}$, i.e. those with genus $g$ and $n$ punctures. Let $\mathscr{Q}_{g, n}$ denote those $\phi$ with at most simple poles at the punctures of $X$. The fiber of $\mathscr{Q}_{g, n}$ over $X \in \mathscr{X}_{g, n}$ is canonically identified with the cotangent space to Teichmüller space at $X$, once a marking is chosen.

Theorem A.3.1. $\mathbf{P} \mathscr{Q}_{\mathrm{g}, \mathrm{n}}$ has compact closure in $\mathbf{P} 2$.

\section{Remarks.}

1. The quadratic differentials compactifying $\mathbf{P} \mathscr{Q}_{\mathbf{g}, n}$ live on the Riemann surfaces compactifying $\mathscr{X}_{\mathrm{g}, n}$, i.e. the plane, the punctured plane and hyperbolic surfaces of smaller complexity. The limiting differentials may have poles of high order at the punctures (although they certainly have no essential singularities.)

2. The fibers of $\mathbf{P} \mathscr{Q}_{g, n} \rightarrow \mathscr{X}_{g, n}$ are projective spaces; in general this is not true for the closure

$$
\overline{\mathbf{P} \mathscr{Q}_{g, n}} \rightarrow \overline{\mathscr{X}_{g, n}}
$$

as we will see in examples.

3. This compactification is related to, but quite distinct from, the compactification of moduli space by Riemann surfaces with nodes and the extension of the cotangent bundle to this completion, discussed in [DM], [Bers], [Masur], [EM] and elsewhere. One might further investigate the analytic or algebraic structure $\overline{\mathbf{P} \mathscr{Q}_{g, m}}$; however its topological structure will be sufficient for our applications.

4. $\mathbf{P} 2$ itself is certainly not compact; for example, in the unit disk with basepoint at $z=0$, the sequence $\left[z^{n} d z^{2}\right]$ has no limit in $\mathbf{P} 2$.

\section{A.3.1. Quadratic differentials from simple closed curves}

To begin the proof, we construct continuous families of quadratic differentials using Poincaré series.

Let $Y$ be a planar Riemann surface with fundamental group $\mathbf{Z}$. Choose an isomorphism

$$
h: Y \rightarrow A(r, R)=\{z: r<|z|<R\} \subset \mathbf{C}^{*},
$$

for $0 \leqq r<R \leqq \infty$, and set $\phi(Y)=h^{*}\left(d z^{2} / z^{2}\right)$. One may check that $\phi(Y)$ is welldefined and varies continuously in $\mathscr{Q}$ as a function of $(Y, w)$ in $\mathscr{X}$. In the $|\phi|$-metric, $Y$ is a flat cylinder of radius 1 . Up to a constant multiple, $\phi(Y)$ is the only quadratic differential on $Y$ invariant under the automorphisms of $Y$. 
Let $(X, v, S) \in \mathscr{S}$ be a Riemann surface together with a finite system of free homotopy classes

$$
S=\left\{\left[\gamma_{1}\right], \ldots,\left[\gamma_{s}\right]\right\},
$$

where $\left[\gamma_{i}\right]$ are conjugacy classes in $\pi_{1}(X)$. The cyclic subgroup generated by $\gamma_{i}$ determines a covering $p_{i}: Y_{i} \rightarrow X$ where $Y_{i}$ is a planar surface with fundamental group $\mathbf{Z}$. Define an associated quadratic differential on $X$ by

$$
\theta(X, v, S)=\sum_{1}^{s}\left(p_{i}\right)_{*} \phi\left(Y_{i}\right)
$$

Proposition A.3.2. The differential $\theta(X, v, S)$ is holomorphic, with at most double poles at the punctures of $X$. Moreover $\theta \neq 0$.

Proof. If $Y_{i}$ is an annulus, $\left|\phi\left(Y_{i}\right)\right|$ is integrable and hence its push-forward is holomorphic with at most simple poles. Otherwise $Y_{i}$ is a punctured plane or punctured disk. Each puncture has a neighborhood mapping injectively to a neighborhood of a puncture on $X$, creating a double pole for $\theta ;|\phi|$ is integrable outside a neighborhood of the punctures, so its push-forward is holomorphic.

To check that $\theta \neq 0$, first suppose that $X$ is a hyperbolic Riemann surface and $S$ is a system of disjoint simple geodesics. Then Wolpert has shown the differentials $p_{*}\left(\phi_{i}\right)$ are linearly independent [Wol, Theorem 3.7]. In fact, there is a perfect pairing between these differentials, though of as elements of the cotangent space to Teichmüller space at $X$, and the Fenchel-Nielsen twist-vector fields for each curve $\left[\gamma_{i}\right]$. (Wolpert's proof is given for compact surfaces, but easily extends to the general situation.)

Otherwise, some element of $S$ represents a peripheral curve around a puncture $p$ of $X$. Then $\theta \neq 0$ because it has a double pole at $p$.

\section{A.3.2. Variation of Poincaré series}

Proposition A.3.3. The mapping $\theta: \mathscr{S} \rightarrow \mathscr{Q}$ associating a quadratic differential to a system of simple closed curves is continuous.

Proof. Let $\left(X_{n}, v_{n}, S_{n}\right) \rightarrow(X, v, S)$. It is easy to reduce to the case $\left|S_{n}\right|=1$; then $S$ consists of one or two curves, depending on whether or not a nonseparating curve is pinching off.

To start, assume $|S|=1$.

Choose basepoints lying over $v_{n} \rightarrow v$ on the corresponding covering spaces, such that $\left(Y_{n}, w_{n}\right) \rightarrow(Y, w)$; then the corresponding quadratic differentials $\phi_{n}$ converge geometrically to $\phi$ on $Y$. To demonstrate that

$$
\theta\left(X_{n}, v_{n}, S_{n}\right) \rightarrow \theta(X, v, S),
$$

it is enough to show that the convergence of $\phi_{n}$ to $\phi$ is fiberwise faithful (by Proposition A.2.5).

We check this case by case. 
1. $X=\mathbf{C}^{*}$.

(a) $X_{n}=\mathrm{C}^{*}$ for all $n$. This case is trivial.

(b) $S_{n}$ are geodesics on hyperbolic surfaces.

In the flat $\left|\phi_{n}\right|$-metric, each $Y_{n}$ is a finite cylinder of fixed circumference whose height is tending to infinity. In the Poincare metric, the thick part of $Y_{n}$ consists of two annuli at the ends of $Y_{n}$ whose $\left|\phi_{n}\right|$-heights are uniformly bounded.
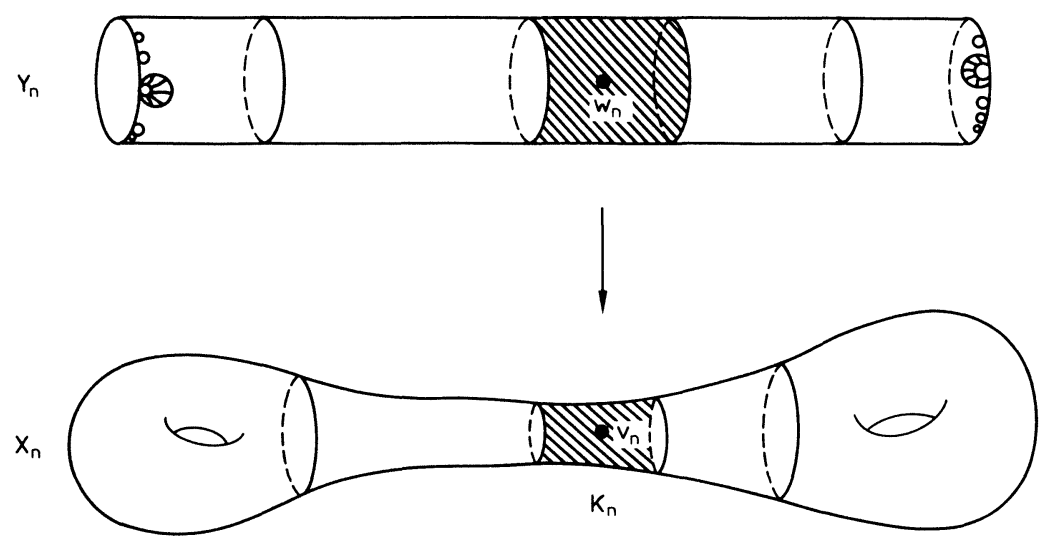

Fig. 4. A short geodesic converging to a cylinder

Let $K_{n} \rightarrow K$ be a faithfully convergence sequence of compact sets on $X_{n} \rightarrow X$. For $n$ large, $K_{n}$ is entirely contained in the component of the thin part corresponding to $S_{n}$, and its pre-image on $Y_{n}$ consists of one component in the thin part (which persists in the limit) and other components in the thick part (near the ends of $Y_{n}$ ); see Fig. 4. The boundary of the thin part on $Y_{n}$ lies over the boundary of the thin part on $X_{n}$, while the Poincare distance from $K_{n}$ to the boundary of the thin part tends to infinity; thus the lifts of $K_{n}$ in the thick part of $Y_{n}$ are confined to a narrow neighborhood of the ends of $Y_{n}$ and their $\left|\phi_{n}\right|$-area tends to zero. Thus $\phi_{n} \rightarrow \phi$ faithfully fiberwise.

(c) $S_{n}$ correspond to punctures on hyperbolic surfaces.

A similar argument applies, with half-infinite cylinders.

2. $X$ is hyperbolic.

(a) $S$ is a geodesic.

The Poincare length $l(S)=\lim l\left(S_{n}\right)$, and $\left|\phi_{n}\right|$-area of $Y_{n}$ is a continuous function of $l\left(S_{n}\right)$, so no mass is lost in the limit and the convergence is faithful.

(b) $S$ is a puncture which is a limit of punctures.

Each $Y_{n}$ is a half-infinite cylinder, such that the portion at fixed $\left|\phi_{n}\right|$-distance from the finite end injects into a neighborhood of the puncture. It follows that the convergence is fiberwise faithful.

(c) $S$ is a puncture which is a limit of separating geodesics.

The thin part corresponding to $S_{n}$ is bounded by a 'near end' and a 'far end'. The distance of a faithfully convergent sequence of compact sets $K_{n}$ from the far end tends to infinity. Each $Y_{n}$ is a finite cylinder, on which the lifts of $K_{n}$ 
to the near end persist in the limit, while those in the far end have small area by the same Poincare metric argument as in case $1 \mathrm{~b}$. Thus the convergence is fiberwise faithful.

Finally, we treat the case $|S|=2$, which we record as:

(d) $S$ is a pair of punctures which are the limit of nonseparating geodesics. Now both ends of the thin part corresponding to $S_{n}$ remain a bounded distance from the basepoint. Let $(Y, w)$ and $\left(Y^{\prime}, w^{\prime}\right)$ denote the two coverings of $X$ corresponding to the two limiting punctures. We may choose basepoints $w_{n}$ and $w_{n}^{\prime}$ near each of the two ends of $Y_{n}$, such that $\left(Y_{n}, w_{n}\right) \rightarrow(Y, w)$ and $\left(Y_{n}, w_{n}^{\prime}\right)$ $\rightarrow\left(Y^{\prime}, w^{\prime}\right)$. Then the pre-images of $K_{n}$ near one end persists on $(Y, w)$, while those in the other end persists on $\left(Y^{\prime}, w^{\prime}\right)$. Since $\theta(X, v, S)$ is defined as the sum of these two Poincaré series, $\theta\left(X_{n}, v_{n}, S_{n}\right) \rightarrow \theta(X, v, S)$ as claimed.

\section{A.3.3. The closure of $\mathbf{P} \mathscr{Q}_{\mathrm{g}, \mathrm{n}}$ is compact}

Proof of Theorem A.3.1. Let $\left(X_{n}, v_{n},\left[\phi_{n}\right]\right)$ be a sequence in $\mathscr{Q}_{g, n}$. We may assume $\left(X_{n}, v_{n}\right) \rightarrow(X, v) \in \overline{\mathscr{X}_{\mathrm{g}, n}}$.

Suppose $X$ is a hyperbolic surface with a simple geodesic whose free homotopy class is $S$. Then we can find corresponding homotopy classes $S_{n}$ on $X_{n}$ such that $\left(X_{n}, v_{n}, S_{n}\right) \rightarrow(X, v, S)$, and hence

$$
\theta_{n}=\theta\left(X_{n}, v_{n}, S_{n}\right) \rightarrow \theta=\theta(X, v, S) .
$$

Each of these differentials is nonzero with at most double poles.

Set $f_{n}=\phi_{n} / \theta_{n}$. Since $\phi_{n}$ has at most simple poles, $f$ is a meromorphic function of degree at most $4 g-4+3 n$. By Theorem A.2.6, there is a sequence of constants $c_{n}$ such that $c_{n} f_{n}$ has a subsequence converging to an invertible function $f$ uniformly on compact subsets of $X-E$, where $E$ is a finite set. Since $\phi_{n}$ is holomorphic, $c_{n} \phi_{n}$ converges geometrically even at $E$, to $f \theta=\phi$. Thus $\left[\phi_{n}\right] \rightarrow[\phi]$ in $\mathbf{P} 2$.

Now suppose $X$ is the punctured plane or the triply punctured sphere. The same argument applies, with $S$ a peripheral curve around each puncture. (It is here we use continuity of $\theta(X, v, S)$ when two punctures are sewn together.)

Finally suppose $X=(\mathbf{C}, 0)$. Rescale $X_{n}$ so its injectivity radius at the basepoint is 1 ; then a subsequence has a limit $\left(X^{\prime}, v^{\prime}, \phi^{\prime}\right)$, since $X^{\prime}$ cannot be a plane. It follows that the unrescaled differentials accumulate on points of the form $\left(\mathbf{C}, 0,\left[\phi(z) d z^{2}\right]\right)$, where $\phi \neq 0$ is a polynomial whose degree is bounded by the order of zero of $\phi$ at the basepoint on $X^{\prime}$.

\section{A.4. Low dimensional examples}

We conclude with some examples of geometric limits.

1. Here is a complete description of the compactification of $\mathbf{P} \mathscr{Q}_{0,4}$, the quadratic differentials on the four-times punctured sphere. First, $\mathscr{X}_{0,4}$ is compactified by spheres with 1,2 or 3 punctures. There is a unique projective class of differen- 
tial with simple poles on a four-times punctured sphere; it has no zeros, so the same is true of the limit. The limiting differentials on a triply-punctured sphere have poles of orders $(1,1,2)$; those on $\mathbf{C}^{*}$ have poles of orders $(1$, 3 ) and $(2,2)$; and the only limit on $\mathbf{C}$ is $\left[d z^{2}\right]$, with a fourth order pole at infinity. The orders of poles determine the projective class uniquely.

Since the poles can be distributed to various punctures, the fibers of the compactification over $\hat{\mathbf{C}}-\{0,1, \infty\}$ and $\mathbf{C}^{*}$ consist of three points, hence are not projective spaces. Certain linear combinations of limiting differentials have zeros, hence do not occur.

2. Consider $\mathbf{P} \mathscr{Q}_{1,2}$, the quadratic differentials on twice-punctured tori. On a compact torus two pairs of points $Z$ and $P$ are the zeros and poles of a quadratic differential iff $Z-P=0$ in the group law (a torus is its own Jacobian).

The four times punctured sphere arises as a limit of twice punctured tori by pinching a non-separating curve; normalize so the two new punctures are at 0 and $\infty$. Then any limiting differential has the form $\phi=f(z) d z^{2} / z^{2}$, where the zeros and poles of $f$ now satisfy $Z / P=1$ in the group law on $C^{*}$ (assuming the divisor of $f$ does not meet 0 or $\infty$.)

The condition $Z / P=1$ is equivalent to the condition that the residues of $\phi$ at 0 and $\infty$ agree.

3. As a final example, we construct a family of differentials on compact surfaces of genus 2. Fix a torus with a holomorphic differential $d z^{2}$. Cut open two segments of the horizontal foliation, each of length $L$, and paste in a cylinder of height $H$ and circumference $2 L$ with its foliation by circles. The result is a quadratic differential on a surface of genus two, with four zeros at the endpoints of the slits.

Suppose the slits are the sides of a square and $L \rightarrow 0$ while $H / L$ is fixed. For a basepoint on the cylinder, the limit differential lives on a punctured torus and has a fourth order pole. Further degenerations yield differentials with poles of order $(4,2,2)$ on $\hat{\mathbf{C}}-\{0,1, \infty\}$, of order $(4,4)$ on $\mathbf{C}^{*}$, and of order 8 on C.

Remark. It would be interesting to have an explicit description of the closure of $\mathbf{P} \mathscr{Q}_{g, n}$ in $\mathbf{P} \mathscr{Q}_{\text {. }}$

The local form of a quadratic differential at a point $p$ is determined by the order $N$ of zero or pole at $p$, and a complex residue $R$ if $N$ is even and $\leqq-2$ (see [Str3]). An implicit description of the compactification can be given by 'regenerating' an element of $\mathbf{P} \mathscr{Q}_{g, n}$. The regeneration is specified by a quadratic differential $\phi$ on a possibly disconnected Riemann surface $X$, plus pairs of punctures (to be glued together) where the orders and residues of $\phi$ are compatible. It seems difficult, however, to describe which $(X, \phi)$ and gluing diagrams actually exist.

\section{References}

[Ba] Baily, W.: Introductory lectures on automorphic forms. Princeton University Press, 1973

[Bers] Bers, L.: Spaces of degenerating Riemann surfaces. In: Discontinuous groups and Riemann surfaces. Ann. Math. Stud. 76, 43-55 (1974) 
[Br] Brooks, R.: The fundamental group and the spectrum of the Laplacian. Comment. Math. Helv. 56, 581-596 (1985)

[Cha] Chabauty, C.: Limites d'ensembles et géometrie des nombers. Bull. Soc. Math. France 78, 143-151 (1950)

[DM] Deligne, P., Mumford, D.: The irreducibility of the space of curves of given genus. Publ. Math., Inst. Hautes Etud. Sci. 36, 75-110 (1969)

[Earle] Earle, C.J.: Some remarks on Poincaré series. Compos. Math. 21, 167-176 (1969)

[EM] Earle, C.J., Marden, A.: Geometric complex coordinates for Teichmüller space. In preparation

[Gar] Gardiner, F.: Teichmüller theory and quadratic differentials. New York: Wiley Interscience 1987

[Gre] Greenleaf, F.P.: Invariant means on topological groups. Van Nostrand 1969

[Grom] Gromov, M.: Structures métriques pour les variétés riemanniennes. CEDIC, Textes mathématiques, 1981

[Har] Harvey, W.J.: Spaces of discrete groups. In: Discrete groups and automorphic forms. New York: Academic Press 1977

[Kra 1] Kra, I.: Automorphic forms and Kleinian groups. New York: W.A. Benjamin, Inc 1972

[Kra 2] Kra, I.: Canonical mappings between Teichmüller spaces. Bull. AMS 4, 143-179 (1981)

[LS] Lyons, T., Sullivan, D.: Function theory, random paths and covering spaces. J. Differ. Geom. 19, 299-323 (1984)

[Masur] Masur, H.: The extension of the Weil-Petersson metric to the boundary of Teichmüller space. Duke Math. J 43, 623-635 (1976)

[Mc] McMullen, C.: Iteration on Teichmüller space. Preprint

[Mil] Milman, D.P.: On some criteria for the regularity of spaces of type (B). Dokl. Akad. Nauk SSSR (N.S.) 20, 243-246 (1938)

[Oh] Ohtake, H.: Lifts of extremal quasiconformal mappings of arbitrary Riemann surfaces. J. Math. Kyoto Univ. 22, 191-200 (1982)

[Pet] Pettis, B.J.: A proof that every uniformly convex space is reflexive. Duke Math. J. 5, 249-253 (1939)

[Pier] Pier, J.P.: Amenable locally compact groups. New York: Wiley Interscience 1984

[Poin] Poincaré, H.: Mémoire sur les fonctions Fuchsiennes. Acta Math. 1, 193-294 (1882/3)

[PS 1] Parson, L.A., Sheingorn, M.: Bounding the norm of the Poincare $\theta$-operator. In: Analytic Number Theory. Lecture Notes Math. Vol. 899. Berlin-Heidelberg-New York: Springer 1981

[PS 2] Parson, L.A., Sheingorn, M.: Exponential sums connected with Ramanujan's function $\tau(n)$. Mathematika 29, 270-277

[Ros] Rosenblatt, J.M.: A generalization of Følner's condition. Math. Scand. 33, 153-170 (1973)

[RS] Reich, E., Strebel, K.: Extremal quasiconformal mappings with given boundary values. In: Contributions to Analysis. New York: Academic Press 1974

[Scott] Scott, P.: Subgroups of surface groups are almost geometric. J. London Math. Soc. 17, $555-565(1978)$

[Str 1] Strebel, K.: On lifts of extremal quasiconformal mappings. J. Anal. Math. 31, 191-203 (1977)

[Str 2] Strebel, K.: On quasiconformal mappings of open Riemann surfaces. Comment. Math. Helv. 53, 301-321 (1978)

[Str 3] Strebel, K.: Quadratic differentials. Berlin-Heidelberg-New York: Springer 1984

[Str4] Strebel, K.: Extremal quasiconformal mappings. MSRI preprint 1986

[Th 1] Thurston, W.P.: Hyperbolic structures on 3-manifolds IV: Construction of hyperbolic manifolds. In preparation

[Th 2] Thurston, W.P.: Geometry and topology of three-manifolds. Princeton lecture notes, 1979

[Wol] Wolpert, S.: The Fenchel-Nielsen deformation. Ann. Math. 115, 501-528 (1982) 
